Lions-type compactness and Rubik actions on the Heisenberg group

Z. M. Balogh and A. Kristály

Research Report 2012-03

30.01.2012

Mathematics Institute

University of Bern

Sidlerstrasse 5

CH-3012 Bern

Switzerland

www.math.unibe.ch 


\title{
Lions-type compactness and Rubik actions on the Heisenberg group
}

\author{
Zoltán M. Balogh ${ }^{1}$ \\ Mathematisches Institut, Universität Bern, Sidlerstrasse 5, 3012 Bern, Switzerland
}

Alexandru Kristály ${ }^{2}$

Babeş-Bolyai University, 400591 Cluj-Napoca, Romania

\begin{abstract}
In this paper we prove a Lions-type compactness embedding result for symmetric unbounded domains of the Heisenberg group. The natural group action on the Heisenberg group $\mathbb{H}^{n}=\mathbb{C}^{n} \times \mathbb{R}$ is provided by the unitary group $U(n) \times\{1\}$ and its appropriate subgroups, which will be used to construct subspaces with specific symmetry and compactness properties in the Folland-Stein's horizontal Sobolev space $H W_{0}^{1,2}\left(\mathbb{H}^{n}\right)$. As an application, we study the multiplicity of solutions for a singular subelliptic problem by exploiting a technique of solving the Rubik-cube applied to subgroups of $U(n) \times\{1\}$. In our approach we employ concentration compactness, group-theoretical arguments, and variational methods.
\end{abstract}

Keywords: Heisenberg group; compact embedding; subelliptic problems; variational methods.

$M S C: 35 \mathrm{R} 03,35 \mathrm{~A} 15$.

\section{Introduction}

It is well-known that compactness of Sobolev embeddings on unbounded domains of $\mathbb{R}^{n}$ can be recovered whenever the domain has appropriate symmetries. This approach is fruitful in the study of variational elliptic problems in the presence of a suitable group action on the Sobolev space. In such cases the principle of symmetric criticality can be applied to the associated energy functional, allowing a variational treatment of the problem. Roughly speaking, if $X$ denotes a Sobolev space where the solutions are being sought, the strategy is to find a topological group $T$, acting continuously on $X$, such that the following two properties simultaneously hold:

- the fixed point set of $X$ with respect to $T$ is an infinite dimensional subspace of $X$ which can be compactly embedded into a suitable Lebesgue space;

- the energy functional associated to the studied problem is $T$-invariant.

In the Euclidean setting, the above approach has been deeply exploited. For instance, if $\Omega=$ $\mathbb{R}^{n}(n \geq 2)$, then the space of radially (resp., spherically) symmetric functions of $H^{1}\left(\mathbb{R}^{n}\right)$ is compactly embedded into $L^{q}\left(\mathbb{R}^{n}\right), q \in\left(2,2^{*}\right)$. Here, the symmetric functions represent the fixed point set of $H^{1}\left(\mathbb{R}^{n}\right)$ with respect to the orthogonal group $T=O(n)$ (resp., $T=O\left(n_{1}\right) \times \ldots \times O\left(n_{l}\right)$, $\left.n=n_{1}+\ldots+n_{l}, n_{i} \geq 2\right)$, see Strauss [20], Lions [14]. A similar argument works for strip-like domains $\Omega=\omega \times \mathbb{R}^{n-m}$, where $\omega \subset \mathbb{R}^{m}$ is bounded and $n-m \geq 2$, obtaining the space of

\footnotetext{
${ }^{1}$ Z. M. Balogh was supported by the Swiss National Science Foundation, the European Science Foundation Project HCAA and the FP7 EU Commission Project CG-DICE.

${ }^{2}$ A. Kristály was supported by a grant of the Romanian National Authority for Scientific Research, CNCSUEFISCDI, project number PN-II-ID-PCE-2011-3-0241, and by the János Bolyai Research Scholarship of the Hungarian Academy of Sciences.
} 
cylindrically symmetric functions on $H_{0}^{1}(\Omega)$ via the group $T=I_{\mathbb{R}^{m}} \times O(n-m)$, see Esteban and Lions [7], Kobayashi and Ôtani [11].

The purpose of the present paper is to develop counterparts of the aforementioned results via appropriate group symmetries on the Heisenberg group $\mathbb{H}^{n}=\mathbb{C}^{n} \times \mathbb{R}(n \geq 1)$ with applications to the theory of singular subelliptic problems defined on unbounded domains of $\mathbb{H}^{n}$. Subelliptic problems involving the Kohn-Laplace operator on unbounded domains of stratified groups have been intensively studied in recent years, see Garofalo and Lanconelli [9], Maad [15], Schindler and Tintarev [19], Tintarev [21]. A persisting assumption for these results was that $\Omega$ is strongly asymptotically contractive. This means that $\Omega \neq \mathbb{H}^{n}$ and for every unbounded sequence $\left\{\eta_{k}\right\} \subset$ $\mathbb{H}^{n}$ there exists a subsequence $\left\{\eta_{k_{j}}\right\}$ such that either

(a) $\mu\left(\liminf \left(\eta_{k_{j}} \circ \Omega\right)\right)=0$, or

(b) there exists a point $\eta_{0} \in \mathbb{H}^{n}$ such that for any $r>0$ there exists an open set $M_{r} \Subset \eta_{0} \circ \Omega$, a closed set $Z$ of measure zero and an integer $j_{r}>0$ such that $\left(\eta_{k_{j}} \circ \Omega\right) \cap B((0,0), r) \subset M_{r} \cup Z$ for every $j>j_{r}$.

Here $\mu(\cdot)$ is the Lebesgue measure, ' lim inf' is the Kuratowski lower-limit, and ' $O$ ' is the usual group operation on $\mathbb{H}^{n}$. Intuitively speaking, strongly asymptotically contractive domains are thin at infinity. For instance [15] shows that if $p \in[0,1]$ and $\Omega_{p}=\left\{(z, t) \in \mathbb{H}^{n}:|t|<1+|z|^{p}\right\}$, then $\Omega_{p}$ is strongly asymptotically contractive if and only if $p \in[0,1)$.

Once a domain $\Omega \subset \mathbb{H}^{n}$ is not strongly asymptotically contractive, $H W_{0}^{1,2}(\Omega)$ need not be compactly embedded into a Lebesgue space. Therefore, in order to obtain compactness, further assumptions are needed which will be formulated in terms of symmetries. Inspired from Tintarev and Fieseler [22], via the concentration compactness principle, in $\S 3$ we state an abstract compactness result for general Carnot groups where a topological group $T$ acts continuously, see Theorem 3.1. We apply this general principle to the Heisenberg group and its natural group action by the unitary group $T=U(n) \times\{1\}$.

To formulate our compactness result, let $\psi_{1}, \psi_{2}:[0, \infty) \rightarrow \mathbb{R}$ be two functions that are bounded on bounded sets, and $\psi_{1}(r)<\psi_{2}(r)$ for every $r \geq 0$. Let

$$
\Omega_{\psi}=\left\{(z, t) \in \mathbb{H}^{n}: \psi_{1}(|z|)<t<\psi_{2}(|z|)\right\},
$$

where $|z|=\sqrt{\left|z_{1}\right|^{2}+\ldots+\left|z_{n}\right|^{2}}$. Our compactness statement reads as follows.

Theorem 1.1 Let $n \geq 1$ and $\Omega_{\psi}$ be from (1.1). Assume that $n=n_{1}+\ldots+n_{l}$ with $n_{i} \geq 1$, $l \geq 1$, and let $T=U\left(n_{1}\right) \times \ldots \times U\left(n_{l}\right) \times\{1\}$. Then

$$
H W_{0, T}^{1,2}\left(\Omega_{\psi}\right)=\left\{u \in H W_{0}^{1,2}\left(\Omega_{\psi}\right): u(z, t)=u(g(z, t)), \forall g \in T\right\},
$$

is compactly embedded into $L^{q}\left(\Omega_{\psi}\right), q \in\left(2,2_{Q}^{*}\right)$.

Here, $Q=2 n+2$ is the homogeneous dimension of $\mathbb{H}^{n}$, while $2_{Q}^{*}=\frac{2 Q}{Q-2}$ is the critical exponent in the Heisenberg group. Note that

$$
H W_{0, T}^{1,2}\left(\Omega_{\psi}\right)=\left\{u \in H W_{0}^{1,2}\left(\Omega_{\psi}\right): u(z, t)=u\left(\left|z_{n_{1}}\right|, \ldots,\left|z_{n_{l}}\right|, t\right), z_{n_{i}} \in \mathbb{C}^{n_{i}}\right\} .
$$

By Theorem 1.1 compactness is induced by symmetries even if the domain $\Omega_{\psi}$ is large at infinity. However, $\Omega_{\psi}$ cannot be "arbitrarily large", i.e., it cannot be replaced by the whole space $\mathbb{H}^{n}$. 
Indeed, the space $H W_{0, T}^{1,2}\left(\mathbb{H}^{n}\right)=\left\{u \in H W_{0}^{1,2}\left(\mathbb{H}^{n}\right): u(z, t)=u\left(\left|z_{n_{1}}\right|, \ldots,\left|z_{n_{l}}\right|, t\right), z_{n_{i}} \in \mathbb{C}^{n_{i}}\right\}$ is not compactly embedded into $L^{q}\left(\mathbb{H}^{n}\right)$. This is due to shiftings along the $t$-direction, see Remark 3.3. A similar phenomenon has been pointed out by Birindelli and Lanconelli [3, Corollary 1.1] concerning De Giorgi's conjecture on Heisenberg groups.

In $\S 4$ we describe symmetrically different functions belonging to $H W_{0}^{1,2}\left(\Omega_{\psi}\right)$ via groups of the type $U\left(n_{1}\right) \times \ldots \times U\left(n_{l}\right) \times\{1\}$ for various splittings of the dimension $n=n_{1}+\ldots+n_{l}$ $\left(n_{i} \geq 1, l \geq 2\right)$. The objective is to find as much mutually different subgroups of $U(n)$ of the form $U\left(n_{1}\right) \times \ldots \times U\left(n_{l}\right)$ as possible such that the group generated by each two of them to act transitively on the unit sphere of $\mathbb{C}^{n}$. In this way, by exploiting a Rubik-cube technique (see Kunkle and Cooperman [13]), we may construct $\left[\frac{n}{2}\right]+1$ subspaces of $H W_{0}^{1,2}\left(\Omega_{\psi}\right)$ which are compactly embedded into $L^{q}\left(\Omega_{\psi}\right), q \in\left(2,2_{Q}^{*}\right)$, and have completely different structures from symmetrical point of view, see Proposition 4.1.

In $\S 5$ we apply the above results to study the singular subelliptic problem

$$
\begin{cases}-\Delta_{\mathbb{H}^{n}} u-\nu V(z, t) u+u=\lambda K(z, t) f(u) & \text { in } \quad \Omega_{\psi} \\ u=0, & \text { on } \partial \Omega_{\psi}\end{cases}
$$

where $\Delta_{\mathbb{H} n}$ is the Kohn-Laplace operator on the Heisenberg group $\mathbb{H}^{n}$, and $\lambda, \nu \geq 0$. We assume that $(0,0) \in \Omega_{\psi}$, and $f \in \mathcal{A}_{q}$ for some $q \in\left(2,2_{Q}^{*}\right)$, where

$$
\mathcal{A}_{q}=\left\{f \in C(\mathbb{R}, \mathbb{R}): \sup _{s \neq 0} \frac{|f(s)|}{|s|+|s|^{q-1}}<\infty\right\} .
$$

On the potentials $V, K: \Omega_{\psi} \rightarrow \mathbb{R}$ we assume:

$\left(H_{V}\right) V$ is measurable, cylindrically symmetric, i.e., $V(z, t)=V(|z|, t)$, and there exists $C_{V}>0$ such that

$$
0 \leq V(z, t) \leq C_{V} \frac{|z|^{2}}{N(z, t)^{4}}, \forall(z, t) \in \Omega_{\psi} \backslash\{(0,0)\} ;
$$

$\left(H_{K}\right) K \in L^{\infty}\left(\Omega_{\psi}\right)$ is cylindrically symmetric.

Two complementary cases will be considered depending on $f: \mathbb{R} \rightarrow \mathbb{R}:$ (a) $f$ is superlinear at infinity, and (b) $f$ is sublinear at infinity.

For the superlinear case, we assume that $f \in \mathcal{A}_{q}$ for some $q \in\left(2,2_{Q}^{*}\right)$. Denoting by $F(s)=$ $\int_{0}^{s} f(t) d t$, we assume:

$\left(f_{1}\right) f(s)=o(|s|)$ as $|s| \rightarrow 0$;

$\left(f_{2}\right)$ there exists $\alpha>2$ such that $s f(s) \geq \alpha F(s)>0$ for all $s \in \mathbb{R} \backslash\{0\}$.

By means of the principle of symmetric criticality and mountain pass arguments, the construction of the symmetrically distinct subspaces provides the following result.

Theorem 1.2 Let $\Omega_{\psi} \subset \mathbb{H}^{n}$ be from (1.1) with $(0,0) \in \Omega_{\psi}, \nu \in\left[0, C_{V}^{-1} n^{2}\right)$ be fixed, and let $V, K: \Omega_{\psi} \rightarrow \mathbb{R}$ be potentials verifying $\left(H_{V}\right)$ and $\left(H_{K}\right)$ with $\inf _{\Omega_{\psi}} K>0$. Let $f \in \mathcal{A}_{q}$ for some $q \in\left(2,2_{Q}^{*}\right)$ verifying $\left(f_{1}\right)$ and $\left(f_{2}\right)$. Then, the following assertions hold: 
(i) Given $T=U\left(n_{1}\right) \times \ldots \times U\left(n_{l}\right) \times\{1\}$ with $n=n_{1}+\ldots+n_{l}$ and $n_{i} \geq 1, l \geq 1$, for every $\lambda>0$, the problem $\left(P_{\lambda}^{\nu}\right)$ has at least a nonzero weak solution in $H W_{0, T}^{1,2}\left(\Omega_{\psi}\right)$;

(ii) In addition, if $f$ is odd, for every $\lambda>0$ problem $\left(P_{\lambda}^{\nu}\right)$ has at least $\left[\frac{n}{2}\right]+1$ sequences of distinct, weak solutions with mutually different symmetric structures.

In the sublinear case, we assume that $f \in C(\mathbb{R}, \mathbb{R})$ verifies

$\left(f_{1}^{\prime}\right) f(s)=o(|s|)$ as $|s| \rightarrow 0 ;$

$\left(f_{2}^{\prime}\right) f(s)=o(|s|)$ as $|s| \rightarrow \infty$;

$\left(f_{3}^{\prime}\right)$ there exists $s_{0} \in \mathbb{R}$ such that $F\left(s_{0}\right)>0$.

We consider the perturbed form of problem $\left(P_{\lambda}^{\nu}\right)$, namely,

$$
\left\{\begin{array}{lll}
-\Delta_{\mathbb{H} n} u-\nu V(z, t) u+u=\lambda K(z, t) f(u)+\theta \tilde{K}(z, t) \tilde{f}(u) & \text { in } \Omega_{\psi}, \\
u=0, & \text { on } \partial \Omega_{\psi}, \quad\left(P_{\lambda, \theta}^{\nu}\right)
\end{array}\right.
$$

and we prove a counterpart of Theorem 1.2 as follows.

Theorem 1.3 Let $\Omega_{\psi} \subset \mathbb{H}^{n}$ be from (1.1) with $(0,0) \in \Omega_{\psi}, \nu \in\left[0, C_{V}^{-1} n^{2}\right)$ be fixed, and let $V, K: \Omega_{\psi} \rightarrow \mathbb{R}$ be potentials verifying $\left(H_{V}\right)$ and $\left(H_{K}\right)$ such that $K \in L^{1}\left(\Omega_{\psi}\right)$ and $\inf _{\omega} K>0$ for some open set $\omega \subset \Omega_{\psi}$. Furthermore, let $f \in C(\mathbb{R}, \mathbb{R})$ be a function verifying $\left(f_{1}^{\prime}\right)-\left(f_{3}^{\prime}\right)$, let $\tilde{f} \in \mathcal{A}_{q}$ for some $q \in\left(2,2_{Q}^{*}\right)$, and $\tilde{K} \in L^{\infty}\left(\Omega_{\psi}\right)$ be a cylindrically symmetric function. Then, the following assertions hold:

(i) For $\lambda \in\left[0, c_{f}^{-1}\|K\|_{L^{\infty}}^{-1}\right)$, problem $\left(P_{\lambda}^{\nu}\right)=\left(P_{\lambda, 0}^{\nu}\right)$ has only the zero solution;

(ii) Given $T=U\left(n_{1}\right) \times \ldots \times U\left(n_{l}\right) \times\{1\}$ with $n=n_{1}+\ldots+n_{l}$ and $n_{i} \geq 1, l \geq 1$, there exists $\lambda^{*}>0$ such that for every $\lambda>\lambda^{*}$, there is $\delta^{\lambda}>0$ with the property that for $\theta \in\left[-\delta^{\lambda}, \delta^{\lambda}\right]$, problem $\left(P_{\lambda, \theta}^{\nu}\right)$ has at least two distinct, nonzero weak solutions in $H W_{0, T}^{1,2}\left(\Omega_{\psi}\right)$;

(iii) In addition, if $f$ and $\tilde{f}$ are odd, there exists $\Lambda^{*}>0$ such that for every $\lambda>\Lambda^{*}$, there is $\delta^{\lambda}>0$ with the property that for $\theta \in\left[-\delta^{\lambda}, \delta^{\lambda}\right]$, the problem $\left(P_{\lambda, \theta}^{\nu}\right)$ has at least $s_{n}=2\left(\left[\frac{n}{2}\right]+1\right)$ distinct pairs of nonzero weak solutions $\left\{ \pm u_{i}^{\lambda, \theta}\right\} \subset H W_{0}^{1,2}\left(\Omega_{\psi}\right), i=1, \ldots, s_{n}$.

The paper is organized as follows. In Section 2 we recall basic notions on stratified groups. Section 3 is devoted to compactness; after formulating a general compactness result for Carnot groups (whose proof is presented in the Appendix), we prove Theorem 1.1. In Section 4 we study Rubik actions on the Heisenberg group $\mathbb{H}^{n}$. In Section 5 we prove Theorems 1.2 and 1.3, respectively.

\section{Preliminaries on stratified groups}

In this section we recall some notions and results from the theory of stratified groups, see Bonfiglioli, Lanconelli and Uguzzoni [5]. A Carnot group is a connected, simply connected, nilpotent Lie group $(G, \circ)$ of dimension at least two (the neutral element being denoted by 0 ) whose Lie algebra $\mathcal{G}$ admits a stratification, i.e., $\mathcal{G}=V_{1} \oplus \ldots \oplus V_{r}$ with $\left[V_{1}, V_{i}\right]=V_{i+1}$ for $i=1, \ldots, r-1$ and $\left[V_{1}, V_{r}\right]=0$. Here, the integer $r$ is called the step of $G$. Let $\langle,\rangle_{0}$ be a fixed inner 
product on $V_{1} \cong \mathbb{R}^{m}$ with associated orthonormal basis $X_{1}, \ldots, X_{m}$. By applying left-translations to these elements on $G$, we introduce the horizontal tangent subbundle of the tangent bundle $T G$ with fibers $\operatorname{span}\left\{X_{1}(p), \ldots, X_{m}(p)\right\}, p \in G$, and extend $\langle,\rangle_{0}$ to the whole $T G$ by group translation. A left-invariant vector field $X$ on $G$ is horizontal if $X(p) \in \operatorname{span}\left\{X_{1}(p), \ldots, X_{m}(p)\right\}$ for every $p \in G$.

We introduce the set of horizontal curves of finite length connecting two arbitrary points $p_{1}, p_{2} \in G$, namely,

$$
H \Gamma_{p_{1}, p_{2}}(G)=\left\{\gamma:[0,1] \rightarrow G: \begin{array}{l}
\gamma \text { is piecewise smooth, } \dot{\gamma}(t) \in V_{1} \text { a.e. } t \in[0,1], \\
\gamma(0)=p_{1}, \gamma(1)=p_{2}, \int_{0}^{1} \sqrt{\langle\dot{\gamma}(t), \dot{\gamma}(t)\rangle_{0}} d t<\infty
\end{array}\right\}
$$

Note that by Chow's theorem, see Gromov [10], $H \Gamma_{p_{1}, p_{2}}(G) \neq \emptyset$, and the Carnot-Carathéodory distance is defined as

$$
d_{C C}\left(p_{1}, p_{2}\right)=\inf \left\{\int_{0}^{1} \sqrt{\langle\dot{\gamma}(t), \dot{\gamma}(t)\rangle_{0}} d t: \gamma \in H \Gamma_{p_{1}, p_{2}}(G)\right\}
$$

which is a left invariant metric on $G$.

For $\lambda>0$ we consider the map $\delta_{\lambda}: \mathcal{G} \rightarrow \mathcal{G}$ by $\delta_{\lambda}(X)=\lambda^{i} X$ for $X \in V_{i}$ which induces an automorphism on $G$ by the exponential map, denoted in the same way. This gives a oneparameter family of anisotropic dilations of $G$ such that $d_{C C}\left(\delta_{\lambda}\left(p_{1}\right), \delta_{\lambda}\left(p_{2}\right)\right)=\lambda d_{C C}\left(p_{1}, p_{2}\right)$ for all $p_{1}, p_{2} \in G$. The Jacobian of $\delta_{\lambda}$ is $\lambda^{Q}$, where the number $Q=\sum_{i=1}^{m} i \operatorname{dim} V_{i}$ is called the homogeneous dimension of $G$. Note that the Haar measure on $G$ is induced by the exponential map from the $k$-dimensional Lebesgue measure, where $\mathcal{G} \cong \mathbb{R}^{k}$ and $k=\sum_{i=1}^{r} \operatorname{dim} V_{i}$; thus, the same notation $\mu$ will be used for both measures. Since $G$ is diffeomorphic with $\mathcal{G} \cong \mathbb{R}^{k}$, one can identify elements $g \in G$ with elements $\left(x_{1}, \ldots, x_{m}, t_{m+1}, \ldots, t_{k}\right) \in \mathbb{R}^{k}$ by $g=\exp \left(\sum_{i=1}^{m} x_{i} X_{i}+\right.$ $\left.\sum_{i=m+1}^{k} t_{i} T_{i}\right)$ where $T_{m+1}, \ldots, T_{k}$ are non-horizontal vectors extending the family $X_{1}, \ldots, X_{m}$ to a basis of $\mathcal{G}$. The horizontal gradient on the Carnot group $G$ is the vector $\nabla_{G}=\left(X_{1}, \ldots, X_{m}\right)$ while the horizontal divergence of a vector field $X=\sum_{i=1}^{m} f_{i} X_{i}+\sum_{i=m+1}^{k} h_{i} T_{i}$ is $\operatorname{div}_{G} X=\sum_{i=1}^{m} X_{i}\left(f_{i}\right)$. In particular, the subelliptic Laplacian (or, Kohn-Laplacian) is defined as $\triangle_{G}=\operatorname{div}_{G} \nabla_{G}=$ $\sum_{i=1}^{m} X_{i}^{2}$.

Let $G_{0} \subset G$ be an open set. The Folland and Stein's horizontal Sobolev space $H W_{0}^{1,2}\left(G_{0}\right)$ is the completion of $C_{0}^{\infty}\left(G_{0}\right)$ with respect to the norm

$$
\|u\|_{H W\left(G_{0}\right)}^{2}=\int_{G_{0}}\left(\sum_{i=1}^{m}\left|X_{i} u\right|_{0}^{2}+u^{2}\right) .
$$

The inner product coming from the $H W\left(G_{0}\right)$-norm will be denoted by $\langle,\rangle_{H W\left(G_{0}\right)}$. It is wellknown that the space $H W_{0}^{1,2}\left(G_{0}\right)$ is continuously embedded into $L^{q}\left(G_{0}\right)$ for every $q \in\left[2,2_{Q}^{*}\right)$, where $2_{Q}^{*}=2 Q /(Q-2)$ when $Q>2$ and $2_{Q}^{*}=\infty$ when $Q=2$, see Folland and Stein [8]. If $G_{0}$ is bounded, the above embedding is compact. Note that $H W^{1,2}(G)=H W_{0}^{1,2}(G)$, and the $H W(G)$-norm from (2.1) is invariant with respect to the left group translations

$$
D_{G}=\left\{g_{\eta}: u \mapsto u \circ \eta, \eta \in G\right\},
$$

where

$$
\left(g_{\eta} u\right)(p)=u(\eta \circ p), p \in G, u \in H W^{1,2}(G) .
$$


It turns out that $\left(H W_{0}^{1,2}(G), D_{G}\right)$ is a dislocation pair in the sense of Tintarev and Fieseler, cf. [22, Proposition 9.1, p. 222], and the elements of $D_{G}$ are unitary operators, i.e., $g_{\eta}^{*}=g_{\eta}^{-1}$.

If the Carnot group $G$ is polarizable in the sense of Balogh and Tyson [2], according to Kombe [12], one has the subelliptic Hardy inequality

$$
\int_{G}\left|\nabla_{G} u\right|_{0}^{2} \geq\left(\frac{Q-2}{2}\right)^{2} \int_{G} \frac{\left|\nabla_{G} N\right|_{0}^{2}}{N^{2}} u^{2}, \quad \forall u \in C_{0}^{\infty}(G),
$$

where $N=u_{2}^{\frac{1}{2-Q}}$ is the homogeneous norm associated to Folland's fundamental solution $u_{2}$ for the sub-Laplacian $\triangle_{G}$. Moreover, the constant $\left(\frac{Q-2}{2}\right)^{2}$ is optimal in (2.3).

Our main example is the Heisenberg group $\mathbb{H}^{n}=\mathbb{C}^{n} \times \mathbb{R}(n \geq 1)$ which is the simplest non-commutative (polarizable) Carnot group with step 2. The group operation is given by

$$
(z, t) \circ\left(z^{\prime}, t^{\prime}\right)=\left(z+z^{\prime}, t+t^{\prime}+2 \operatorname{Im}\left\langle z, z^{\prime}\right\rangle\right),
$$

where $z=\left(z_{1}, \ldots, z_{n}\right) \in \mathbb{C}^{n}, t \in \mathbb{R}$, and $\left\langle z, z^{\prime}\right\rangle=\sum_{j=1}^{n} z_{j} \overline{z_{j}^{\prime}}$ is the Hermitian inner product. Denoting by $z_{j}=x_{j}+i y_{j}$, then $\left(x_{1}, \ldots, x_{n}, y_{1}, \ldots, y_{n}, t\right)$ form a real coordinate system for $\mathbb{H}^{n}$ and the system of vector fields

$$
\begin{aligned}
& X_{j}^{1}=\partial_{x_{j}}+2 y_{j} \partial_{t}, \\
& X_{j}^{2}=\partial_{y_{j}}-2 x_{j} \partial_{t}, \\
& T=\partial_{t},
\end{aligned}
$$

form a basis for the left invariant vector fields of $\mathbb{H}^{n}$. Its Lie algebra has the stratification $\mathcal{H}_{n}=V_{1} \oplus V_{2}$ where the $2 n$-dimensional horizontal space $V_{1}$ is spanned by $\left\{X_{j}^{1}, X_{j}^{2}\right\}_{j=1, \ldots, n}$, while $V_{2}$ is spanned by $T$. The homogeneous dimension of $\mathbb{H}^{n}$ is $Q=2 n+2$, thus the best constant $\left(\frac{Q-2}{2}\right)^{2}$ in $(2.3)$ for $G=\mathbb{H}^{n}$ becomes $n^{2}$. The $(2 n+1)$-dimensional Lebesgue measure $\mu(\cdot)$ on $\mathbb{H}^{n}$ is a Haar measure of the group.

Let $N(z, t)=\left(|z|^{4}+t^{2}\right)^{\frac{1}{4}}$ be the gauge norm on $\mathbb{H}^{n}$, and the Korányi metric $d_{K}\left((z, t),\left(z^{\prime}, t^{\prime}\right)\right)=$ $N\left(\left(z^{\prime}, t^{\prime}\right)^{-1} \circ(z, t)\right)$. It is well-known that $d_{C C}$ and $d_{K}$ are equivalent metrics on $\mathbb{H}^{n}$. The Korányi ball of center $\left(z_{0}, t_{0}\right) \in \mathbb{H}^{n}$ and radius $r>0$ is $B\left(\left(z_{0}, t_{0}\right), r\right)=\left\{(z, t) \in \mathbb{H}^{n}\right.$ : $\left.d_{K}\left((z, t),\left(z_{0}, t_{0}\right)\right)<r\right\}$. A simple calculation shows that $\mu(B((z, t), r))=r^{Q} \mu(B((0,0), 1))$, and $\left|\nabla_{\mathbb{H}^{n}} N(z, t)\right|_{0}=\frac{|z|}{N(z, t)},(z, t) \in \mathbb{H}^{n} \backslash\{(0,0)\}$.

\section{Compact embeddings on stratified groups via symmetries}

Let $(G, \circ)$ be a Carnot group, and $(T, \cdot)$ be a closed topological group with neutral element $e$. We say that $T$ acts continuously on $G, T * G \mapsto G$, if

(TG0) $e * p=p$ for all $p \in G$;

(TG1) $\hat{\tau}_{1} *\left(\hat{\tau}_{2} * p\right)=\left(\hat{\tau}_{1} \cdot \hat{\tau}_{2}\right) * p$ for all $\hat{\tau}_{1}, \hat{\tau}_{2} \in T$ and $p \in G$,

and left-distributively if

(TG2) $\hat{\tau} *\left(p_{1} \circ p_{2}\right)=\left(\hat{\tau} * p_{1}\right) \circ\left(\hat{\tau} * p_{2}\right)$ for all $\hat{\tau} \in T$ and $p_{1}, p_{2} \in G$. 
A set $G_{0} \subset G$ is $T$-invariant, if $T * G_{0}=G_{0}$, i.e., $\hat{\tau} * p \in G_{0}$ for every $\hat{\tau} \in T$ and $p \in G_{0}$.

We shall assume that $T$ induces an action on $H W_{0}^{1,2}(G), T \# H W_{0}^{1,2}(G) \mapsto H W_{0}^{1,2}(G)$, by

$$
(\hat{\tau} \# u)(p)=u\left(\hat{\tau}^{-1} * p\right) .
$$

Once (TG0) and (TG1) hold, the action of $T$ on $H W_{0}^{1,2}(G)$ is continuous. The group $T$ acts isometrically on $H W_{0}^{1,2}(G)$ if

$$
\|\hat{\tau} \# u\|_{H W(G)}=\|u\|_{H W(G)} \text { for all } \hat{\tau} \in T, u \in H W_{0}^{1,2}(G) .
$$

Let $G_{0}$ be an open subset of $G$, and assume that

$(H)_{T}^{G_{0}}$ : For every $\left\{\eta_{k}\right\} \subset G$ such that $d_{C C}\left(0, \eta_{k}\right) \rightarrow \infty$ and $\mu\left(\lim \inf \left(\eta_{k} \circ G_{0}\right)\right)>0$, there exists a subsequence $\left\{\eta_{k_{j}}\right\}$ of $\left\{\eta_{k}\right\}$ and a subgroup $T_{\left\{\eta_{k_{j}}\right\}}$ of $T$ such that card $\left(T_{\left\{\eta_{k_{j}}\right\}}\right)=\infty$ and for all $\hat{\tau}_{1}, \hat{\tau}_{2} \in T_{\left\{\eta_{k_{j}}\right\}}, \hat{\tau}_{1} \neq \hat{\tau}_{2}$, one has

$$
\lim _{j \rightarrow \infty} \inf _{p \in G} d_{C C}\left(\left(\hat{\tau}_{1} * \eta_{k_{j}}\right) \circ p,\left(\hat{\tau}_{2} * \eta_{k_{j}}\right) \circ p\right)=\infty .
$$

Hypothesis $(H)_{T}^{G_{0}}$ can be viewed as a replacement of the strongly asymptotically contractiveness of $G_{0}$. Indeed, while a strongly asymptotically contractive domain is thin at infinity, hypothesis $(H)_{T}^{G_{0}}$ allows to deal with a class of domains which are large at infinity. In the sequel, we state an abstract compactness result for Carnot groups whenever $(H)_{T}^{G_{0}}$ holds.

Theorem 3.1 Let $(G, \circ)$ be a Carnot group of homogeneous dimension $Q \geq 2,(T, \cdot)$ be a closed infinite topological group acting continuously and left-distributively on $G$. Assume that $T$ acts isometrically on $H W_{0}^{1,2}(G)$ by (3.1). Let $G_{0}$ be a T-invariant open subset of $G$ and assume that $(H)_{T}^{G_{0}}$ holds. Then,

$$
H W_{0, T}^{1,2}\left(G_{0}\right)=\left\{u \in H W_{0}^{1,2}\left(G_{0}\right): \hat{\tau} \# u=u, \forall \hat{\tau} \in T\right\}
$$

is compactly embedded into $L^{q}\left(G_{0}\right)$ for every $q \in\left(2,2_{Q}^{*}\right)$.

Remark 3.1 We shall apply this general Lions-type theorem to the Heisenberg group $G=\mathbb{H}^{n}$ where $T$ is the action of the unitary group $U(n) \times\{1\}$ on $\mathbb{H}^{n}$. This statement is strongly related to the results of Tintarev and Fieseler [22] who considered the case of group actions $T$ by translations. The proof of Theorem 3.1 follows the ideas from [22]. For the sake of completeness we present it in the Appendix.

Recall that the unitary group is

$$
U(n)=U(n ; \mathbb{C})=\left\{\tau \in G L(n ; \mathbb{C}):\left\langle\tau z, \tau z^{\prime}\right\rangle=\left\langle z, z^{\prime}\right\rangle \text { for all } z, z^{\prime} \in \mathbb{C}^{n}\right\}
$$

where $\langle$,$\rangle denotes the standard Hermitian inner product.$

Let $T=U(n) \times\{1\}$ be the group with its natural multiplication law ' $'$, and we introduce the action $T * \mathbb{H}^{n} \mapsto \mathbb{H}^{n}$ as

$$
\hat{\tau} *(z, t)=(\tau z, t) \quad \text { for } \hat{\tau}=(\tau, 1) \in T \text { and }(z, t) \in \mathbb{H}^{n} .
$$


Lemma 3.1 The group $(T, \cdot)=(U(n) \times\{1\}, \cdot)$ acts continuously and left-distributively on $\left(\mathbb{H}^{n}, \mathrm{o}\right)$ via the action (3.3), i.e., (TG0)-(TG2) hold.

Proof. (TG0) and (TG1) hold trivially. The definition of the unitary group $U(n)$ gives

$$
\begin{aligned}
\left(\hat{\tau} *\left(z_{1}, t_{1}\right)\right) \circ\left(\hat{\tau} *\left(z_{2}, t_{2}\right)\right) & =\left(\tau z_{1}, t_{1}\right) \circ\left(\tau z_{2}, t_{2}\right) \\
& =\left(\tau z_{1}+\tau z_{2}, t_{1}+t_{2}+2 \operatorname{Im}\left\langle\tau z_{1}, \tau z_{2}\right\rangle\right) \\
& =\left(\tau\left(z_{1}+z_{2}\right), t_{1}+t_{2}+2 \operatorname{Im}\left\langle z_{1}, z_{2}\right\rangle\right) \\
& =\hat{\tau} *\left(\left(z_{1}, t_{1}\right) \circ\left(z_{2}, t_{2}\right)\right),
\end{aligned}
$$

which proves (TG2).

The following observation seems to be known to specialists; since we were not able to find a reference, we include its proof for the sake of completeness.

Lemma 3.2 The group $(T, \cdot)=(U(n) \times\{1\}, \cdot)$ acts isometrically on $H W_{0}^{1,2}\left(\mathbb{H}^{n}\right)$ by $(3.1)$.

Proof. We prove that

$$
\|\hat{\tau} \# u\|_{H W\left(\mathbb{H}^{n}\right)}=\|u\|_{H W\left(\mathbb{H}^{n}\right)}, \quad \forall \hat{\tau}=(\tau, 1) \in T, u \in H W_{0}^{1,2}\left(\mathbb{H}^{n}\right),
$$

where the operation ' $\#$ ' is given by (3.1). To check (3.4), let $A(z, t)=A(x, y, t)$ be the $(2 n+$ $1) \times(2 n+1)$ symmetric matrix with elements $a_{i j}=\delta_{i j}$ if $i, j=1, \ldots, 2 n ; a_{(2 n+1) j}=2 y_{j}$ if $j=1, \ldots, n ; a_{(2 n+1) j}=-2 x_{j}$ if $j=n+1, \ldots, 2 n$; and $a_{(2 n+1)(2 n+1)}=4|z|^{2}$. In other words, $A(z, t)=\left(\begin{array}{cc}I_{\mathbb{R}^{2 n}} & J z \\ (J z)^{T} & 4|z|^{2}\end{array}\right)$, where $J=\left(\begin{array}{cc}0 & 2 I_{\mathbb{R}^{n}} \\ -2 I_{\mathbb{R}^{n}} & 0\end{array}\right)$ is the symplectic matrix. Note that

$$
\int_{\mathbb{H}^{n}}\left|\nabla_{\mathbb{H}^{n}} u\right|_{0}^{2} d z d t=\int_{\mathbb{H}^{n}}\langle A(z, t) \nabla u(z, t), \nabla u(z, t)\rangle d z d t,
$$

where $\langle$,$\rangle is the inner product in \mathbb{R}^{2 n+1}$ and $\nabla$ is the Euclidean gradient. In order to prove (3.4), it is enough to check that

$$
\int_{\mathbb{H}^{n}}\langle A(z, t) \nabla v(z, t), \nabla v(z, t)\rangle d z d t=\int_{\mathbb{H}^{n}}\langle A(z, t) \nabla u(z, t), \nabla u(z, t)\rangle d z d t,
$$

where $v(z, t)=(\hat{\tau} \# u)(z, t)=u\left(\tau^{-1} z, t\right)$. Since

$$
\nabla v(z, t)=\left(\hat{\tau}^{-1}\right)^{T} \nabla u\left(\tau^{-1} z, t\right),
$$

where $\left(\hat{\tau}^{-1}\right)^{T}$ denotes the transpose of $\hat{\tau}^{-1}$, the last relation becomes

$$
\int_{\mathbb{H}^{n}}\left\langle A(z, t)\left(\hat{\tau}^{-1}\right)^{T} \nabla u\left(\tau^{-1} z, t\right),\left(\hat{\tau}^{-1}\right)^{T} \nabla u\left(\tau^{-1} z, t\right)\right\rangle d z d t=\int_{\mathbb{H}^{n}}\langle A(z, t) \nabla u(z, t), \nabla u(z, t)\rangle d z d t,
$$

that is

$$
\int_{\mathbb{H}^{n}}\left\langle\hat{\tau}^{-1} A(z, t)\left(\hat{\tau}^{-1}\right)^{T} \nabla u\left(\tau^{-1} z, t\right), \nabla u\left(\tau^{-1} z, t\right)\right\rangle d z d t=\int_{\mathbb{H}^{n}}\langle A(z, t) \nabla u(z, t), \nabla u(z, t)\rangle d z d t .
$$


Changing the variable $z$ to $\tau z$ in the first integral (and keeping in mind that the Jacobian has determinant 1), our claim is concluded once we prove that

$$
\hat{\tau}^{-1} A(\tau z, t)\left(\hat{\tau}^{-1}\right)^{T}=A(z, t) .
$$

First, one has

$$
\begin{aligned}
\hat{\tau}^{-1} A(\tau z, t)\left(\hat{\tau}^{-1}\right)^{T} & =\left(\begin{array}{cc}
\tau^{-1} & 0 \\
0 & 1
\end{array}\right) \cdot\left(\begin{array}{cc}
I_{\mathbb{R}^{2 n}} & J(\tau z) \\
(J(\tau z))^{T} & 4|\tau z|^{2}
\end{array}\right) \cdot\left(\begin{array}{cc}
\left(\tau^{-1}\right)^{T} & 0 \\
0 & 1
\end{array}\right) \\
& =\left(\begin{array}{cc}
\tau^{-1}\left(\tau^{-1}\right)^{T} & \tau^{-1} J(\tau z) \\
(J(\tau z))^{T}\left(\tau^{-1}\right)^{T} & 4|\tau z|^{2}
\end{array}\right) .
\end{aligned}
$$

Then, since $\tau \in U(n)=O(2 n) \cap G L(n ; \mathbb{C}) \cap S p(2 n ; \mathbb{R})$, we have that $\tau^{-1}\left(\tau^{-1}\right)^{T}=I_{\mathbb{R}^{2 n}}$ and $\tau^{-1} J \tau=J$, which proves our claim, thus (3.4).

Remark 3.2 The above argument actually shows that the structure of the unitary group is indispensable in the following sense: $\tau \in G L(n ; \mathbb{C})$ verifies relation $(3.5)$ for every $(z, t) \in \mathbb{H}^{n}$ if and only if $\tau \in U(n)$. Roughly speaking, from 'invariance' point of view, the unitary groups play the same role in the Heisenberg setting as the orthogonal groups in the Euclidean framework.

Proof of Theorem 1.1. We are going to apply Theorem 3.1 with $(G, \circ)=\left(\mathbb{H}^{n}, \circ\right), T=U\left(n_{1}\right) \times$ $\ldots \times U\left(n_{l}\right) \times\{1\}$, and $G_{0}=\Omega_{\psi}$. In view of Lemmas $3.1 \& 3.2$, it remains to verify $(H)_{T}^{G_{0}}$.

Let $\eta_{k}=\left(z_{k}, t_{k}\right) \in \mathbb{C}^{n} \times \mathbb{R}=\mathbb{H}^{n}$, and assume that the sequence $\left\{\eta_{k}\right\}$ is unbounded with the property $\mu\left(\lim \inf \left(\eta_{k} \circ \Omega_{\psi}\right)\right)>0$. We claim that $\left\{z_{k}\right\}$ is unbounded. By contradiction, we assume that $\left\{z_{k}\right\} \subset \mathbb{C}^{n}$ is bounded; consequently, $\left\{t_{k}\right\} \subset \mathbb{R}$ should be unbounded. Fix $i \in \mathbb{N}$, and let $A_{i}=\cap_{k \geq i}\left(\eta_{k} \circ \Omega_{\psi}\right)$. Then,

$$
\begin{aligned}
\left(z^{\prime}, t^{\prime}\right) \in A_{i} & \Leftrightarrow\left(z^{\prime}, t^{\prime}\right) \in \eta_{k} \circ \Omega_{\psi}, \forall k \geq i \\
& \Leftrightarrow \eta_{k}^{-1} \circ\left(z^{\prime}, t^{\prime}\right) \in \Omega_{\psi}, \forall k \geq i \\
& \Leftrightarrow\left(z^{\prime}-z_{k}, t^{\prime}-t_{k}-2 \operatorname{Im}\left\langle z_{k}, z^{\prime}\right\rangle\right) \in \Omega_{\psi}, \forall k \geq i \\
& \Leftrightarrow \psi_{1}\left(\left|z^{\prime}-z_{k}\right|\right)<t^{\prime}-t_{k}-2 \operatorname{Im}\left\langle z_{k}, z^{\prime}\right\rangle<\psi_{2}\left(\left|z^{\prime}-z_{k}\right|\right), \quad \forall k \geq i .
\end{aligned}
$$

Since $\left\{z^{\prime}-z_{k}\right\}$ is bounded and the functions $\psi_{1}$ and $\psi_{2}$ map bounded sets into bounded sets, the sequence $\left\{t^{\prime}-t_{k}-2 \operatorname{Im}\left\langle z_{k}, z^{\prime}\right\rangle\right\} \in \mathbb{R}$ is bounded as well, which contradicts the unboundedness of $\left\{t_{k}\right\}$. Consequently, $A_{i}=\emptyset$, so $\liminf \left(\eta_{k} \circ \Omega_{\psi}\right)=\cup_{i \geq 1} A_{i}=\emptyset$, a contradiction with the assumption. Therefore, the sequence $\left\{z_{k}\right\} \subset \mathbb{C}^{n}$ is unbounded, as claimed above.

If $z_{k}=\left(z_{k}^{n_{1}}, \ldots, z_{k}^{n_{l}}\right)$ with $z_{k}^{n_{i}} \in \mathbb{C}^{n_{i}}$, we can choose $i_{0} \in\{1, \ldots, l\}$ and $j_{0} \in\left\{1, \ldots, n_{i_{0}}\right\}$ such that a subsequence $\left\{z_{k_{j}}^{n_{i_{0}}, j_{0}}\right\}$ of $\left\{z_{k}^{n_{i_{0}}, j_{0}}\right\} \subset \mathbb{C}$ is unbounded, where $z_{k}^{n_{i_{0}}}=\left(z_{k}^{n_{i_{0}}, 1}, \ldots, z_{k}^{n_{i_{0}}, n_{i_{0}}}\right)$. Let $T_{\left\{\eta_{k_{j}}\right\}}$ be a subgroup of $T$ defined by the $S^{1}$-action in the unbounded component $z_{k_{j}}^{n_{i_{0}}, j_{0}}$ of $z_{k_{j}}$, where $S^{1}=\left\{e^{i \phi}: \phi \in[0,2 \pi)\right\}$ is the circle group. With the above constructions in our mind, we may choose

$$
T_{\left\{\eta_{k_{j}}\right\}}=I_{\mathbb{C}^{n_{1}+\ldots+n_{i_{0}-1}+j_{0}-1}} \times S^{1} \times I_{\mathbb{C}^{-j_{0}+n_{i_{0}}+\ldots+n_{l}}} \times\{1\} .
$$

It is clear that $T_{\left\{\eta_{k_{j}}\right\}}$ is a closed subgroup of $T=U\left(n_{1}\right) \times \ldots \times U\left(n_{l}\right) \times\{1\}$, and for every

$$
\hat{\tau}_{k}=\left(\tau_{k}, 1\right)=I_{\mathbb{C}^{n_{1}+\ldots+n_{i_{0}-1}+j_{0}-1}} \times \tau_{k}^{\prime} \times I_{\mathbb{C}^{-j_{0}+n_{i_{0}}+\ldots+n_{l}}} \times\{1\} \in T_{\left\{\eta_{k_{j}}\right\}}
$$


with $\tau_{k}^{\prime}=\cos \phi_{k}+i \sin \phi_{k}, \phi_{k} \in[0,2 \pi), k=1,2$ and $\phi_{1} \neq \phi_{2}$, it yields that

$$
\begin{aligned}
\inf _{p \in \mathbb{H}^{n}} d_{K}\left(\left(\hat{\tau}_{1} * \eta_{k_{j}}\right) \circ p,\left(\hat{\tau}_{2} * \eta_{k_{j}}\right) \circ p\right) & =\inf _{p \in \mathbb{H}^{n}} N\left((-p) \circ\left(-\tau_{2} z_{k_{j}},-t_{k_{j}}\right) \circ\left(\tau_{1} z_{k_{j}}, t_{k_{j}}\right) \circ p\right) \\
& \geq\left|\tau_{2}^{\prime} z_{k_{j}}^{n_{i_{0}}, j_{0}}-\tau_{1}^{\prime} z_{k_{j}}^{n_{i_{0}}, j_{0}}\right| \\
& =\left[2-2 \cos \left(\phi_{2}-\phi_{1}\right)\right]^{\frac{1}{2}}\left|z_{k_{j}}^{n_{i_{0}}, j_{0}}\right| \rightarrow \infty,
\end{aligned}
$$

as $j \rightarrow \infty$. Since $d_{C C}$ is an equivalent metric with $d_{K}$, relation (3.2) is verified. The conclusion follows immediately.

If $T=U(n) \times\{1\}$ in Theorem 1.1, the following can be stated:

Corollary 3.1 Let $\Omega_{\psi}$ be from (1.1). Then, the space of cylindrically symmetric functions of $H W_{0}^{1,2}\left(\Omega_{\psi}\right)$, i.e.,

$$
H W_{0, \text { cyl }}^{1,2}\left(\Omega_{\psi}\right)=\left\{u \in H W_{0}^{1,2}\left(\Omega_{\psi}\right): u(z, t)=u(|z|, t)\right\},
$$

is compactly embedded into $L^{q}\left(\Omega_{\psi}\right), q \in\left(2,2_{Q}^{*}\right)$.

Remark 3.3 The domain $\Omega_{\psi}$ cannot be replaced by the whole space $\mathbb{H}^{n}$, i.e., the space

$$
H W_{0, T}^{1,2}\left(\mathbb{H}^{n}\right)=\left\{u \in H W^{1,2}\left(\mathbb{H}^{n}\right): u(z, t)=u\left(\left|z_{n_{1}}\right|, \ldots,\left|z_{n_{l}}\right|, t\right), z_{n_{i}} \in \mathbb{C}^{n_{i}}\right\}
$$

is not compactly embedded into $L^{q}\left(\mathbb{H}^{n}\right), n=n_{1}+\ldots+n_{l}$ with $n_{i} \geq 1, l \geq 1$. Indeed, let $u_{0}(z, t)=1+\cos N(z, t)$ when $N(z, t) \leq \pi$, and $u_{0}(z, t)=0$ when $N(z, t) \geq \pi$. Then, the sequence $u_{k}(z, t)=u_{0}(z, t-k)$ is bounded in $H W_{0, T}^{1,2}\left(\mathbb{H}^{n}\right)$, it converges weakly to 0 , but $u_{k} \nrightarrow 0$ in $L^{q}\left(\mathbb{H}^{n}\right)$ for any $q \in\left(2,2_{Q}^{*}\right)$ since $\left\|u_{k}\right\|_{L^{q}}=\left\|u_{0}\right\|_{L^{q}} \neq 0$ for every $k \in \mathbb{N}$. As we can see, the lack of compactness of embedding of $H W_{0, T}^{1,2}\left(\mathbb{H}^{n}\right)$ into $L^{q}\left(\mathbb{H}^{n}\right)$ comes from the possibility of translations along the whole $t$-direction, which is not the case for $H W_{0, T}^{1,2}\left(\Omega_{\psi}\right)$. This example also shows the indispensability of the central hypothesis $(H)_{T}^{G_{0}}$ from Theorem 3.1. For instance, if $\eta_{k}=(0, k) \in \mathbb{H}^{n}$, then $d_{C C}\left(0, \eta_{k}\right) \rightarrow \infty$ and $\mu\left(\liminf \left(\eta_{k} \circ \mathbb{H}^{n}\right)\right)=\infty$; however, for every $p \in \mathbb{H}^{n}$ and $\hat{\tau}_{k}=\left(\tau_{k}, 1\right) \in U(n) \times\{1\}, k=1,2$, with $\tau_{1} \neq \tau_{2}$, one has

$$
d_{C C}\left(\left(\hat{\tau}_{1} * \eta_{k}\right) \circ p,\left(\hat{\tau}_{2} * \eta_{k}\right) \circ p\right)=d_{C C}\left(\eta_{k} \circ p, \eta_{k} \circ p\right)=0,
$$

i.e., relation (3.2) fails.

Remark 3.4 If the functions $\psi_{i}(i=1,2)$ are bounded, the domain $\Omega_{\psi}$ is strongly asymptotically contractive. In this case, not only $H W_{0, T}^{1,2}\left(\Omega_{\psi}\right)$ but also $H W_{0}^{1,2}\left(\Omega_{\psi}\right)$ can be compactly embedded into $L^{q}\left(\Omega_{\psi}\right), q \in\left(2,2_{Q}^{*}\right)$, see Garofalo and Lanconelli [9], Schindler and Tintarev [19].

\section{Rubik actions and symmetries}

In the previous subsection we proved that the subgroup $U\left(n_{1}\right) \times \ldots \times U\left(n_{l}\right)$ of the unitary group $U(n)$ (with $n=n_{1}+\ldots+n_{l}$ ) produces the compact embedding of $T$-invariant functions of $H W_{0}^{1,2}\left(\Omega_{\psi}\right)$ into $L^{q}\left(\Omega_{\psi}\right)$ where $T=U\left(n_{1}\right) \times \ldots \times U\left(n_{l}\right) \times\{1\}$ and $q \in\left(2,2_{Q}^{*}\right)$. The main 
purpose of the present section is to describe symmetrical differences of functions belonging to $H W_{0}^{1,2}\left(\Omega_{\psi}\right)$ via subgroups of the type $U\left(n_{1}\right) \times \ldots \times U\left(n_{l}\right)$ for various splittings of the dimension $n$. In order to solve this question we exploit a Rubik-cube technique. Roughly speaking, the space dimension $n$ corresponds to the number of faces of the cube, while the sides of the cube are certain blocks in the splitting group $U\left(n_{1}\right) \times \ldots \times U\left(n_{l}\right)$. If we consider only one copy of such a proper splitting, the Rubik-cube cannot be solved/restored because only some specific moves are allowed, thus there is a lack of transitivity on the cube. However, combining appropriate splittings simultaneously, different moves complete each other recovering the transitivity, thus solving the cube. The precise construction is described in the sequel.

\subsection{Transitivity of combined Rubik-type moves on subgroups of $U(n)$}

Let $n \geq 2$ and for $i \in\left\{1, \ldots,\left[\frac{n}{2}\right]\right\}$ we consider the subgroup of the unitary group $U(n)$ :

$$
T_{n, i}= \begin{cases}U\left(\frac{n}{2}\right) \times U\left(\frac{n}{2}\right), & \text { if } \quad n=2 i, \\ U(i) \times U(n-2 i) \times U(i), & \text { if } \quad n \neq 2 i .\end{cases}
$$

In the sequel, $\left[T_{n, i} ; T_{n, j}\right]$ will denote the group generated by $T_{n, i}$ and $T_{n, j}$. Although $T_{n, i}$ does not act transitively on the sphere $S^{2 n-1}=\left\{z \in \mathbb{C}^{n}:|z|=1\right\}$, we have

Lemma 4.1 Let $i, j \in\left\{1, \ldots,\left[\frac{n}{2}\right]\right\}$ with $i \neq j$. Then the group $\left[T_{n, i} ; T_{n, j}\right]$ acts transitively on the sphere $S^{2 n-1}$. Moreover, for every $z_{1}, z_{2} \in S^{2 n-1}$ there exists $\tau \in\left[T_{n, i} ; T_{n, j}\right]$ such that $z_{1}=\tau z_{2}$ and $\tau$ is the composition of at most 3 alternating elements from $T_{n, i}$ and $T_{n, j}$, starting with an element from $T_{n, \max \{i, j\}}$.

Proof. For simplicity, set $0_{k}=(0, \ldots, 0) \in \mathbb{C}^{k}=\mathbb{R}^{2 k}, k \in\{1, \ldots, n\}$. We may assume that $j>i$. Fix $z=\left(z_{1}, z_{2}, z_{3}\right) \in S^{2 n-1}$ arbitrarily with $z_{1}, z_{3} \in \mathbb{C}^{j}$ and $z_{2} \in \mathbb{C}^{n-2 j}$. [If $j=n / 2$, the term $z_{2}$ simply disappears from $z$.] Since $U(j)$ acts transitively on $S^{2 j-1}$, one can find $\tau_{j}^{1}, \tau_{j}^{2} \in U(j)$ such that if $\tau_{j}=\tau_{j}^{1} \times I_{\mathbb{C}^{n-2 j}} \times \tau_{j}^{2} \in T_{n, j}$, then

$$
\tau_{j} z=\left(0_{j-1},\left|z_{1}\right|, z_{2},\left|z_{3}\right|, 0_{j-1}\right) .
$$

Now, we switch to the action with an element from $T_{n, i}$. Since $j-1 \geq i$, due to the transitivity of $U(n-2 i)$ on $S^{2 n-4 i-1}$, there exists $\tau_{i}^{1} \in U(n-2 i)$ such that

$$
\tau_{i}^{1}\left(0_{j-i-1},\left|z_{1}\right|, z_{2},\left|z_{3}\right|, 0_{j-i-1}\right)=\left(1,0_{n-2 i-1}\right) .
$$

Therefore, if $\tau_{i}=I_{\mathbb{C}^{i}} \times \tau_{i}^{1} \times I_{\mathbb{C}^{i}} \in T_{n, i}$ then

$$
\tau_{i} \tau_{j} z=\left(0_{i}, 1,0_{n-i-1}\right) .
$$

Now, repeating the above argument for another element $\tilde{z} \in S^{2 n-1}$, one can find $\tilde{\tau}_{i} \in T_{n, i}$ and $\tilde{\tau}_{j} \in T_{n, j}$ such that

$$
\tilde{\tau}_{i} \tilde{\tau}_{j} \tilde{z}=\left(0_{i}, 1,0_{n-i-1}\right)
$$

Thus,

$$
z=\tau_{j}^{-1} \tau_{i}^{-1} \tilde{\tau}_{i} \tilde{\tau}_{j} \tilde{z}=\tau_{j}^{-1} \bar{\tau}_{i} \tilde{\tau}_{j} \tilde{z}
$$

where $\bar{\tau}_{i}=\tau_{i}^{-1} \tilde{\tau}_{i} \in T_{n, i}$. 


\subsection{Symmetrically distinct elements of $H W_{0}^{1,2}\left(\Omega_{\psi}\right)$}

Let $n \geq 2$ be fixed. For every $i \in\left\{1, \ldots,\left[\frac{n}{2}\right]\right\}$, we consider the matrix $\zeta_{i}$ as

$$
\zeta_{i}=\left(\begin{array}{cc}
0 & I_{\mathbb{C}^{\frac{n}{2}}} \\
I_{\mathbb{C}^{\frac{n}{2}}} & 0
\end{array}\right) \text { if } n=2 i \text {, and } \zeta_{i}=\left(\begin{array}{ccc}
0 & 0 & I_{\mathbb{C}^{i}} \\
0 & I_{\mathbb{C}^{n-2 i}} & 0 \\
I_{\mathbb{C}^{i}} & 0 & 0
\end{array}\right) \text { if } n \neq 2 i
$$

A simple verification shows that $\zeta_{i} \in U(n) \backslash T_{n, i}, \zeta_{i}^{2}=I_{\mathbb{C}^{n}}$, and $\zeta_{i} T_{n, i} \zeta_{i}^{-1}=T_{n, i}$.

In the sequel, we will follow a construction from Bartsch and Willem [4]. Let $\hat{T}_{n, i}^{\zeta_{i}}$ be the group generated by $\hat{T}_{n, i}=T_{n, i} \times\{1\}$ and $\hat{\zeta}_{i}=\left(\zeta_{i}, 1\right)$. On account of the above properties, the group generated by $\hat{T}_{n, i}$ and $\hat{\zeta}_{i}$ is

$$
\hat{T}_{n, i}^{\zeta_{i}} \stackrel{\text { def }}{=}\left[\hat{T}_{n, i} ; \hat{\zeta}_{i}\right]=\hat{T}_{n, i} \cup \hat{\zeta}_{i} \hat{T}_{n, i}
$$

i.e., only two types of elements in $\hat{T}_{n, i}^{\zeta_{i}}$ can be distinguished; namely, elements of the form $\hat{\tau} \in \hat{T}_{n, i}$, and $\hat{\zeta}_{i} \hat{\tau} \in \hat{T}_{n, i}^{\zeta_{i}} \backslash \hat{T}_{n, i}$ (with $\left.\hat{\tau} \in \hat{T}_{n, i}\right)$. The action of the group $\hat{T}_{n, i}^{\zeta_{i}}$ on $H W_{0}^{1,2}\left(\Omega_{\psi}\right)$ is defined by

$$
(\tilde{\tau} \# u)(z, t)= \begin{cases}u\left(\hat{\tau}^{-1} *(z, t)\right) & \text { if } \quad \tilde{\tau}=\hat{\tau} \in \hat{T}_{n, i} \\ -u\left(\left(\hat{\zeta}_{i} \hat{\tau}\right)^{-1} *(z, t)\right) & \text { if } \quad \tilde{\tau}=\hat{\zeta}_{i} \hat{\tau} \in \hat{T}_{n, i}^{\zeta_{i}} \backslash \hat{T}_{n, i}\end{cases}
$$

for $\tilde{\tau} \in \hat{T}_{n, i}^{\zeta_{i}}, u \in H W_{0}^{1,2}\left(\Omega_{\psi}\right)$ and $(z, t) \in \Omega_{\psi}$, where ${ }^{\prime} *^{\prime}$ comes from (3.3).

The following result provides a precise information on the mutually symmetric differences for the spaces of $\hat{T}_{n, i}^{\zeta_{i}}$-invariant functions in $H W_{0}^{1,2}\left(\Omega_{\psi}\right)$.

Proposition 4.1 Let $n \geq 2$ and $\Omega_{\psi}$ be from (1.1). The following statements hold true:

(i) The space

$$
H W_{0, \hat{T}_{n, i}^{\zeta_{i}}}^{1,2}\left(\Omega_{\psi}\right)=\left\{u \in H W_{0}^{1,2}\left(\Omega_{\psi}\right): \tilde{\tau} \# u=u, \forall \tilde{\tau} \in \hat{T}_{n, i}^{\zeta_{i}}\right\},
$$

where ' $\#^{\prime}$ is given in (4.2), is compactly embedded into $L^{q}\left(\Omega_{\psi}\right)$ for all $q \in\left(2,2_{Q}^{*}\right)$ and $i \in\left\{1, \ldots,\left[\frac{n}{2}\right]\right\}$;

(ii) $H W_{0, \hat{T}_{n, i}^{\zeta_{i}}}^{1,2}\left(\Omega_{\psi}\right) \cap H W_{0, \mathrm{cyl}}^{1,2}\left(\Omega_{\psi}\right)=\{0\}$ for all $i \in\left\{1, \ldots,\left[\frac{n}{2}\right]\right\}$;

(iii) If $n \geq 4$, then $H W_{0, \hat{T}_{n, i}^{\zeta_{i}}}^{1,2}\left(\Omega_{\psi}\right) \cap H W_{0, \hat{T}_{n, j}^{\zeta_{j}}}^{1,2}\left(\Omega_{\psi}\right)=\{0\}$ for all $i, j \in\left\{1, \ldots,\left[\frac{n}{2}\right]\right\}, i \neq j$.

Proof. (i) On the one hand, the first relation of (4.2) implies that $H W_{0, \hat{T}_{n, i}^{\zeta_{i}}}^{1,2}\left(\Omega_{\psi}\right) \subset H W_{0, \hat{T}_{n, i}}^{1,2}\left(\Omega_{\psi}\right)$. On the other hand, on account of Theorem 1.1, the space $H W_{0, \hat{T}_{n, i}}^{1,2}\left(\Omega_{\psi}\right)$ is compactly embedded into $L^{q}\left(\Omega_{\psi}\right), q \in\left(2,2_{Q}^{*}\right)$.

(ii) Let us fix $u \in H W_{0, \hat{T}_{n, i}^{\zeta_{i}}}^{1,2}\left(\Omega_{\psi}\right) \cap H W_{0, \mathrm{cyl}}^{1,2}\left(\Omega_{\psi}\right)$. Since $u$ is $\hat{T}_{n, i}^{\zeta_{i}}$-invariant, the second relation from (4.2) implies in particular that $u(z, t)=-u\left(\hat{\zeta}_{i}^{-1} *(z, t)\right)=-u\left(\zeta_{i}^{-1} z, t\right)$ for every $(z, t) \in \Omega_{\psi}$. Since $u$ is cylindrically symmetric, i.e., $u(z, t)=u(|z|, t)$, and $|z|=\left|\zeta_{i}^{-1} z\right|$, we necessarily have that $u=0$. 
(iii) Let $u \in H W_{0, \hat{T}_{n, i}^{\zeta_{i}}}^{1,2}\left(\Omega_{\psi}\right) \cap H W_{0, \hat{T}_{n, j}^{\zeta_{j}}}^{1,2}\left(\Omega_{\psi}\right)$. Note that the latter fact means in particular that $u$ is both $\hat{T}_{n, i}-$, and $\hat{T}_{n, j}$-invariant, thus invariant with respect to $\left[T_{n, i} ; T_{n, j}\right] \times\{1\}$. Since $\left[T_{n, i} ; T_{n, j}\right]$ acts transitively on $S^{2 n-1}$ by Lemma 4.1, it possesses only a single group orbit. This means that $u$ is actually a cylindrically symmetric function on $\Omega_{\psi}$, thus $u=0$ from (ii).

\section{$5 \quad$ Proof of Theorems 1.2 and 1.3}

For $f \in \mathcal{A}_{q}$, let $F(s)=\int_{0}^{s} f(t) d t$. Fix $\nu \in\left[0, C_{V}^{-1} n^{2}\right)$. For every $\lambda>0$, we introduce the energy functional $\mathcal{E}_{\lambda}: H W_{0}^{1,2}\left(\Omega_{\psi}\right) \rightarrow \mathbb{R}$ associated with problem $\left(P_{\lambda}^{\nu}\right)$, namely,

$$
\mathcal{E}_{\lambda}(u)=\frac{1}{2}\|u\|_{H W\left(\Omega_{\psi}\right)}^{2}-\frac{\nu}{2} \int_{\Omega_{\psi}} V(z, t) u^{2} d z d t-\lambda \mathcal{F}(u),
$$

where

$$
\mathcal{F}(u)=\int_{\Omega_{\psi}} K(z, t) F(u(z, t)) d z d t .
$$

For the sake of simplicity of notations, we do not mention the parameter $\nu$ in the functional $\mathcal{E}_{\lambda}$. Since $f \in \mathcal{A}_{q}$ for some $q \in\left(2,2_{Q}^{*}\right)$, on account of $\left(H_{V}\right),\left(H_{K}\right)$ and subelliptic Hardy inequality (see (2.3)), the functional $\mathcal{E}_{\lambda}$ is well-defined, of class $C^{1}$ and its critical points are precisely the weak solutions for $\left(P_{\lambda}^{\nu}\right)$. Moreover, since $\nu \in\left[0, C_{V}^{-1} n^{2}\right)$, the norm $\|\cdot\|_{H W\left(\Omega_{\psi}\right)}$ is equivalent with the norm given by

$$
\|u\|_{\nu}=\left(\|u\|_{H W\left(\Omega_{\psi}\right)}^{2}-\nu \int_{\Omega_{\psi}} V(z, t) u^{2} d z d t\right)^{1 / 2} .
$$

First, we prove Theorem 1.2. Note that hypothesis $\left(f_{2}\right)$ is the standard Ambrosetti and Rabinowitz assumption (see [1]), which implies that for some $s_{0}>0$ and $c>0$, one has $|f(s)| \geq c|s|^{\alpha-1}$ for all $|s|>s_{0}$, i.e., $f$ is superlinear at infinity.

Proof of Theorem 1.2. (i) Fix $\lambda>0$. Let $\mathcal{E}_{\lambda}^{T}$ be the restriction of the energy functional $\mathcal{E}_{\lambda}$ to the space $H W_{0, T}^{1,2}\left(\Omega_{\psi}\right)$. On account of Theorem 1.1 and hypotheses $\left(f_{1}\right),\left(f_{2}\right)$, one can apply in a standard manner the mountain pass theorem of Ambrosetti and Rabinowitz [1] to $\mathcal{E}_{\lambda}^{T}$, obtaining a critical point $u_{\lambda} \in H W_{0, T}^{1,2}\left(\Omega_{\psi}\right)$ of $\mathcal{E}_{\lambda}^{T}$ with positive energy-level; in particular, $u_{\lambda} \neq 0$. Due to relation (3.4) and the cylindrical symmetry of $V$ and $K$, the functional $\mathcal{E}_{\lambda}$ is $T$-invariant where the action of $T$ on $H W_{0}^{1,2}\left(\Omega_{\psi}\right)$ is given by (3.1). Now, the principle of symmetric criticality of Palais [16] implies that $u_{\lambda}$ is a critical point also for $\mathcal{E}_{\lambda}$, thus a weak solution for $\left(P_{\lambda}^{\nu}\right)$.

(ii) Let $n \geq 2$. First, since $V$ and $K$ are cylindrically symmetric, the functional $\mathcal{E}_{\lambda}$ is $U(n) \times\{1\}$-invariant with respect to the action defined by (3.1). Second, since $f$ is odd, $\mathcal{E}_{\lambda}$ is an even functional, thus $\mathcal{E}_{\lambda}$ is $\hat{T}_{n, i}^{\zeta_{i}}$-invariant with respect to the action from (4.2). Let $\mathcal{E}_{\lambda}^{i}$ $\left(i=1, \ldots,\left[\frac{n}{2}\right]\right)$ and $\mathcal{E}_{\lambda}^{\text {cyl }}$ be the restrictions of $\mathcal{E}_{\lambda}$ to the spaces $H W_{0, \hat{T}_{n, i}^{\zeta_{i}}}^{1,2}\left(\Omega_{\psi}\right)$ and $H W_{0, \text { cyl }}^{1,2}\left(\Omega_{\psi}\right)$, respectively. By exploiting Proposition 4.1 (i) and Corollary 3.1 as well as hypotheses $\left(f_{1}\right)$, $\left(f_{2}\right)$, we can apply the symmetric version of the mountain pass theorem to $\mathcal{E}_{\lambda}^{i}\left(i=1, \ldots,\left[\frac{n}{2}\right]\right)$ and $\mathcal{E}_{\lambda}^{\text {cyl }}$, respectively, see e.g. Willem [23, Theorem 3.6]. Therefore, one can guarantee the existence of the sequences of distinct critical points $\left\{u_{k}^{\lambda, i}\right\} \subset H W_{0, \hat{T}_{n, i}^{\zeta_{i}}}^{1,2}\left(\Omega_{\psi}\right)\left(i=1, \ldots,\left[\frac{n}{2}\right]\right)$ and $\left\{u_{k}^{\lambda}\right\} \subset H W_{0, \text { cyl }}^{1,2}\left(\Omega_{\psi}\right)$ of the functionals $\mathcal{E}_{\lambda}^{i}\left(i=1, \ldots,\left[\frac{n}{2}\right]\right)$ and $\mathcal{E}_{\lambda}^{\text {cyl }}$, respectively. They are also 
critical points of $\mathcal{E}_{\lambda}$ due to the principle of symmetric criticality. In view of Proposition 4.1 (ii) \& (iii), the symmetric structure of the elements in the aforementioned sequences mutually differ.

Before proving Theorem 1.3 some remarks are in order on the assumptions $\left(f_{1}^{\prime}\right)-\left(f_{3}^{\prime}\right)$.

Remark 5.1 (a) Hypotheses $\left(f_{1}\right)$ and $\left(f_{1}^{\prime}\right)$ coincide, which means that $f$ is superlinear at the origin. Hypothesis $\left(f_{2}^{\prime}\right)$ is a counterpart of the superlinearity assumption $\left(f_{2}\right)$. Due to $\left(f_{1}^{\prime}\right)$ and $\left(f_{2}^{\prime}\right)$, we have $f \in \mathcal{A}_{q}$ for every $q \in\left(2,2_{Q}^{*}\right)$. These hypotheses also imply that

$$
\lim _{s \rightarrow 0} \frac{F(s)}{s^{2}}=\lim _{|s| \rightarrow \infty} \frac{F(s)}{s^{2}}=0 .
$$

Moreover, if $K \in L^{\infty}\left(\Omega_{\psi}\right) \cap L^{1}\left(\Omega_{\psi}\right)$, a simple verification shows that $\mathcal{F}$ defined in (5.1) inherits similar properties as $F$, i.e.,

$$
\begin{aligned}
& \lim _{\|u\|_{\nu} \rightarrow 0} \frac{\mathcal{F}(u)}{\|u\|_{\nu}^{2}}=0 \\
& \lim _{\|u\|_{\nu} \rightarrow \infty} \frac{\mathcal{F}(u)}{\|u\|_{\nu}^{2}}=0
\end{aligned}
$$

where $\|\cdot\|_{\nu}$ is defined in (5.2).

(b) The number $c_{f}=\max _{s \neq 0} \frac{|f(s)|}{|s|}$ is well-defined and positive.

(c) If $X$ is a closed subspace of $H W_{0}^{1,2}\left(\Omega_{\psi}\right)$ which is compactly embedded into $L^{q}\left(\Omega_{\psi}\right)$, $q \in\left(2,2_{Q}^{*}\right)$, then $\left.\mathcal{F}\right|_{X}$ has a compact derivative.

Remark 5.2 Let us keep the notations from the proof of Theorem 1.2 and assume that $\left(f_{1}^{\prime}\right)-$ $\left(f_{3}^{\prime}\right)$ hold. Then, 0 is a local minimum point for the functionals $\mathcal{E}_{\lambda}^{T}$ and $\mathcal{E}_{\lambda}^{i}\left(i=1, \ldots,\left[\frac{n}{2}\right]\right)$, cf. (5.3). Moreover, these functionals are coercive (cf. (5.4)), bounded from bellow, satisfying the Palais-Smale condition; thus, all of them have a global minimum point with negative energy-level for large values of $\lambda$. Consequently, the well-known critical point theorem of Pucci and Serrin [17] gives a third critical point for these functionals. Summing up, for large values of $\lambda>0$, one can expect the existence of at least two nonzero weak solutions for $\left(P_{\lambda}^{\nu}\right)$ in $H W_{0, T}^{1,2}\left(\Omega_{\psi}\right)$, and at least $2\left(\left[\frac{n}{2}\right]+1\right)$ nonzero weak solutions for $\left(P_{\lambda}^{\nu}\right)$ whenever $f$ is odd.

Theorem 1.3 gives a more precise information as the one stated in Remark 5.2; indeed, it shows that the number of solutions described below is stable with respect to small subcritical perturbations. In order to prove it, we recall a result established by Ricceri [18].

If $X$ is a Banach space, we denote by $\mathcal{W}_{X}$ the class of those functionals $E: X \rightarrow \mathbb{R}$ having the property that if $\left\{u_{k}\right\}$ is a sequence in $X$ converging weakly to $u \in X$ and $\liminf _{k \rightarrow \infty} E\left(u_{k}\right) \leq$ $E(u)$ then $\left\{u_{k}\right\}$ has a subsequence converging strongly to $u$.

Theorem 5.1 [18, Theorem 2] Let $(X,\|\cdot\|)$ be a separable, reflexive, real Banach space, let $E_{1}: X \rightarrow \mathbb{R}$ be a coercive, sequentially weakly lower semicontinuous $C^{1}$ functional belonging to $\mathcal{W}_{X}$, bounded on each bounded subset of $X$ and whose derivative admits a continuous inverse on $X^{*}$. Let $E_{2}: X \rightarrow \mathbb{R}$ be a $C^{1}$ functional with compact derivative. Assume that $E_{1}$ has a strict local minimum point $u_{0}$ with $E_{1}\left(u_{0}\right)=E_{2}\left(u_{0}\right)=0$. 
Assume that $\varrho<\chi$, where

$$
\begin{gathered}
\varrho:=\max \left\{0, \limsup _{\|u\| \rightarrow \infty} \frac{E_{2}(u)}{E_{1}(u)}, \limsup _{u \rightarrow u_{0}} \frac{E_{2}(u)}{E_{1}(u)}\right\}, \\
\chi=\sup _{E_{1}(u)>0} \frac{E_{2}(u)}{E_{1}(u)} .
\end{gathered}
$$

Then, for each compact interval $[a, b] \subset(1 / \chi, 1 / \varrho)$ (with the conventions $1 / 0=\infty$ and $1 / \infty=0$ ) there exists $\kappa>0$ with the following property: for every $\lambda \in[a, b]$ and every $C^{1}$ functional $E_{3}: X \rightarrow \mathbb{R}$ with compact derivative, there exists $\delta>0$ such that for each $\theta \in[-\delta, \delta]$, the equation $E_{1}^{\prime}(u)-\lambda E_{2}^{\prime}(u)-\theta E_{3}^{\prime}(u)=0$ admits at least three solutions in $X$ having norm less than $\kappa$.

Proof of Theorem 1.3. (i) Let $u \in H W_{0}^{1,2}\left(\Omega_{\psi}\right)$ be a solution of $\left(P_{\lambda}^{\nu}\right)$. Multiplying $\left(P_{\lambda}^{\nu}\right)$ by $u$, using the Green theorem, the subelliptic Hardy inequality (2.3) with hypothesis $\left(H_{V}\right)$, the fact that $\nu \in\left[0, C_{V}^{-1} n^{2}\right.$ ), and the definition of number $c_{f}>0$ (see Remark 5.1(b)), we obtain that

$$
\begin{aligned}
\int_{\Omega_{\psi}} u^{2} & \leq \int_{\Omega_{\psi}}\left(\left|\nabla_{\mathbb{H}^{n}} u\right|_{0}^{2}-\nu V(z, t) u^{2}+u^{2}\right) d z d t \\
& =\lambda \int_{\Omega_{\psi}} K(z, t) f(u) u d z d t \\
& \leq \lambda\|K\|_{L^{\infty}} c_{f} \int_{\Omega_{\psi}} u^{2} .
\end{aligned}
$$

If $0 \leq \lambda<c_{f}^{-1}\|K\|_{L^{\infty}}^{-1}$, the above estimate implies $u=0$.

In the sequel, we are going to prove (ii) and (iii) by applying Theorem 5.1. First, let $\hat{\omega}=\cup\{\hat{\tau} \omega: \hat{\tau}=(\tau, 1), \tau \in U(n)\}$, where the set $\omega$ is from the hypothesis of the theorem. Since $K$ is cylindrically symmetric, one has

$$
\inf _{\hat{\omega}} K=\inf _{\omega} K>0 .
$$

Moreover, one can find $\left(z_{0}, t_{0}\right) \in \Omega_{\psi}$ and $R>0$ such that

$$
R<2\left|z_{0}\right|(\sqrt{2}-1)
$$

and

$$
A_{R}=\left\{(z, t) \in \mathbb{H}^{n}:|| z|-| z_{0}|| \leq R,\left|t-t_{0}\right| \leq R\right\} \subset \hat{\omega} .
$$

Clearly, for every $\sigma \in(0,1]$, one has $A_{\sigma R} \subset A_{R} \subset \hat{\omega}$ and $\mu\left(A_{\sigma R}\right)>0$.

(ii) Let $T=U\left(n_{1}\right) \times \ldots \times U\left(n_{l}\right) \times\{1\}$ with $n=n_{1}+\ldots+n_{l}$ and $n_{i} \geq 1, l \geq 1$. We are going to apply Theorem 5.1 with the choices $X=H W_{0, T}^{1,2}\left(\Omega_{\psi}\right)$ and $E_{1}, E_{2}, E_{3}: H W_{0, T}^{1,2}\left(\Omega_{\psi}\right) \rightarrow \mathbb{R}$ which are the restrictions of $\frac{1}{2}\|\cdot\|_{\nu}^{2}, \mathcal{F}$ and $\tilde{\mathcal{F}}$ to the space $H W_{0, T}^{1,2}\left(\Omega_{\psi}\right)$, respectively, where $\tilde{\mathcal{F}}(u)=\int_{\Omega_{\psi}} \tilde{K}(z, t) \tilde{F}(u) d z d t, u \in H W_{0}^{1,2}\left(\Omega_{\psi}\right)$. Note that as a norm-type functional, $E_{1}$ is coercive, sequentially weakly lower semicontinuous, it belongs to $\mathcal{W}_{H W_{0, T}^{1,2}\left(\Omega_{\psi}\right)}$, it is bounded on each bounded subset of $H W_{0, T}^{1,2}\left(\Omega_{\psi}\right)$ and its derivative admits a continuous inverse on the 
dual space of $H W_{0, T}^{1,2}\left(\Omega_{\psi}\right)$. On account of Remark 5.1(c) and Theorem 1.1, $E_{2}$ and $E_{3}$ are $C^{1}$ functionals with compact derivative. Moreover, $u_{0}=0$ is a strict global minimum point of $E_{1}$, $E_{1}(0)=E_{2}(0)=0$, and (5.3) and (5.4) yield $\varrho=0$ (see relation (5.5)). In the sequel, we shall prove that

$$
\chi=\sup \left\{\frac{2 \mathcal{F}(u)}{\|u\|_{\nu}^{2}}: u \in H W_{0, T}^{1,2}\left(\Omega_{\psi}\right) \backslash\{0\}\right\} \in(0, \infty) .
$$

Let $s_{0} \in \mathbb{R}$ be the number from $\left(f_{3}^{\prime}\right)$. For every $\sigma \in(0,1)$ we consider the truncation function $u_{\sigma}: \Omega_{\psi} \rightarrow \mathbb{R}$ defined by

$$
u_{\sigma}(z, t)=\frac{s_{0}}{1-\sigma}\left(1-\max \left(\frac{|| z|-| z_{0}||}{R}, \frac{\left|t-t_{0}\right|}{R}, \sigma\right)\right)_{+}
$$

where $r_{+}=\max (r, 0)$. It is clear that $u_{\sigma} \in H W_{0, \text { cyl }}^{1,2}\left(\Omega_{\psi}\right) \subset H W_{0, T}^{1,2}\left(\Omega_{\psi}\right)$ and

(p1) $\operatorname{supp} u_{\sigma}=A_{R}$;

(p2) $\left\|u_{\sigma}\right\|_{L^{\infty}} \leq\left|s_{0}\right|$;

(p3) $u_{\sigma}(z, t)=s_{0}$ for every $(z, t) \in A_{\sigma R}$.

The above properties, the subelliptic Hardy inequality $(2.3)$, and hypotheses $\left(H_{V}\right)$ and $\left(H_{K}\right)$ imply that

$$
\left\|u_{\sigma}\right\|_{\nu}^{2} \geq \int_{\Omega_{\psi}} u_{\sigma}^{2} \geq s_{0}^{2} \mu\left(A_{\sigma R}\right)
$$

and

$$
\begin{aligned}
\mathcal{F}\left(u_{\sigma}\right) & =\int_{A_{R}} K(z, t) F\left(u_{\sigma}(z, t)\right) d z d t \\
& =\int_{A_{\sigma R}} K(z, t) F\left(u_{\sigma}(z, t)\right) d z d t+\int_{A_{R} \backslash A_{\sigma R}} K(z, t) F\left(u_{\sigma}(z, t)\right) d z d t \\
& \geq \inf _{A_{\sigma R}} K \cdot F\left(s_{0}\right) \mu\left(A_{\sigma R}\right)-\|K\|_{L^{\infty}} \max _{|t| \leq\left|s_{0}\right|}|F(t)| \mu\left(A_{R} \backslash A_{\sigma R}\right) .
\end{aligned}
$$

If $\sigma \rightarrow 1$, the right-hand sides of the above expressions are positive. Consequently, from (5.3) and (5.4),

$$
\chi=\sup \left\{\frac{2 \mathcal{F}(u)}{\|u\|_{\nu}^{2}}: u \in H W_{0, T}^{1,2}\left(\Omega_{\psi}\right) \backslash\{0\}\right\} \in(0, \infty)
$$

and the number

$$
\lambda^{*}=\inf \left\{\frac{\|u\|_{\nu}^{2}}{2 \mathcal{F}(u)}: u \in H W_{0, T}^{1,2}\left(\Omega_{\psi}\right), \mathcal{F}(u)>0\right\}<\infty
$$

is well-defined. Moreover, one has $\chi^{-1}=\lambda^{*}$.

Applying Theorem 5.1, for every $\lambda>\lambda^{*}=\chi^{-1}>0$, there exists $\delta^{\lambda}>0$ such that for each $\theta \in\left[-\delta^{\lambda}, \delta^{\lambda}\right]$, the functional $E_{1}-\lambda E_{2}-\theta E_{3}$ has at least three critical points in $H W_{0, T}^{1,2}\left(\Omega_{\psi}\right)$. Since the functional $\mathcal{E}_{\lambda, \theta}=\frac{1}{2}\|\cdot\|_{\nu}^{2}-\lambda \mathcal{F}-\theta \tilde{\mathcal{F}}$ is $T$-invariant where the action of $T$ on $H W_{0}^{1,2}\left(\Omega_{\psi}\right)$ is given by (3.1), the principle of symmetric criticality implies that the critical points of $E_{1}-\lambda E_{2}-\theta E_{3}$ are also critical points for $\mathcal{E}_{\lambda, \theta}$, thus weak solutions for $\left(P_{\lambda, \theta}^{\nu}\right)$. 
(iii) If $n=1$, the claim easily follows after a suitable modification of the proof of (ii); here, the energy functional $\mathcal{E}_{\lambda, \theta}=\frac{1}{2}\|\cdot\|_{\nu}^{2}-\lambda \mathcal{F}-\theta \tilde{\mathcal{F}}$ is even, thus the solutions appear in pairs which belong to $H W_{0, \text { cyl }}^{1,2}\left(\Omega_{\psi}\right)$. Now, let $n \geq 2$, and fix $i \in\left\{1, \ldots,\left[\frac{n}{2}\right]\right\}$ arbitrarily. The difficulty relies on the construction of a suitable truncation function in $H W_{0, \hat{T}_{n, i}^{\zeta_{i}}}^{1,2}\left(\Omega_{\psi}\right)$ with properties similar to (p1)-(p3). To complete this aim, we first introduce the auxiliary function $e_{i}: \mathbb{C}^{i} \times \mathbb{C}^{i} \times \mathbb{R} \rightarrow \mathbb{R}$ by

$$
e_{i}(z, \tilde{z}, t)=\frac{2}{R} \sqrt{\left(|z|-\left|z_{0}\right|+\frac{R}{2}\right)^{2}+|\tilde{z}|^{2}+\left(t-t_{0}\right)^{2}}
$$

where $z_{0}, t_{0}$ and $R>0$ are from (5.8) and (5.9). We also introduce the sets

$$
S_{1}=\left\{(z, \tilde{z}, t) \in \mathbb{C}^{i} \times \mathbb{C}^{i} \times \mathbb{R}: e_{i}(z, \tilde{z}, t) \leq 1\right\}
$$

and

$$
S_{2}=\left\{(z, \tilde{z}, t) \in \mathbb{C}^{i} \times \mathbb{C}^{i} \times \mathbb{R}: e_{i}(\tilde{z}, z, t) \leq 1\right\} .
$$

A simple reasoning based on (5.8) shows that

$$
S_{1} \cap S_{2}=\emptyset .
$$

For every $\sigma \in(0,1]$, we introduce the set in $\mathbb{H}^{n}$ by

$$
S_{\sigma}^{i}= \begin{cases}\left\{\left(z_{1}, z_{2}, t\right) \in \mathbb{C}^{i} \times \mathbb{C}^{i} \times \mathbb{R}: e_{i}\left(z_{1}, z_{2}, t\right) \leq \sigma \text { or } e_{i}\left(z_{2}, z_{1}, t\right) \leq \sigma\right\}, & \text { if } n=2 i, \\
\left\{\left(z_{1}, z_{2}, z_{3}, t\right) \in \mathbb{C}^{i} \times \mathbb{C}^{n-2 i} \times \mathbb{C}^{i} \times \mathbb{R}: \begin{array}{l}
e_{i}\left(z_{1}, z_{3}, t\right) \leq \sigma \text { or } e_{i}\left(z_{3}, z_{1}, t\right) \leq \sigma, \\
\text { and }\left|z_{2}\right| \leq \frac{\sigma R}{2}
\end{array}\right\}, & \text { if } \quad n \neq 2 i .\end{cases}
$$

It is clear that the set $S_{\sigma}^{i}$ is $\hat{T}_{n, i}^{\zeta_{i}}$-invariant (that is, $\tilde{\tau} S_{\sigma}^{i} \subset S_{\sigma}^{i}$ for every $\tilde{\tau} \in \hat{T}_{n, i}^{\zeta_{i}}$ ), $S_{\sigma}^{i} \subseteq S_{1}^{i}$, $\mu\left(S_{\sigma}^{i}\right)>0$ for every $\sigma \in(0,1]$ and

$$
\lim _{\sigma \rightarrow 1} \mu\left(S_{1}^{i} \backslash S_{\sigma}^{i}\right)=0
$$

Now, we prove that

$$
S_{1}^{i} \subset A_{R}
$$

We consider that $n \neq 2 i$, the case $n=2 i$ is similar. Let $\left(z_{1}, z_{2}, z_{3}, t\right) \in S_{1}^{i}$ such that $e_{i}\left(z_{1}, z_{3}, t\right) \leq$ 1 and $\left|z_{2}\right| \leq \frac{R}{2}$. In particular, the first inequality implies that $\left|t-t_{0}\right| \leq \frac{R}{2}$ and $\left(\left|z_{0}\right|-R\right)^{2} \leq$ $\left|z_{1}\right|^{2}+\left|z_{3}\right|^{2} \leq\left|z_{0}\right|^{2}$. Consequently,

$$
\begin{gathered}
|z|^{2}=\left|z_{1}\right|^{2}+\left|z_{2}\right|^{2}+\left|z_{3}\right|^{2} \leq\left|z_{0}\right|^{2}+\left(\frac{R}{2}\right)^{2}<\left(\left|z_{0}\right|+R\right)^{2}, \\
|z|^{2}=\left|z_{1}\right|^{2}+\left|z_{2}\right|^{2}+\left|z_{3}\right|^{2} \geq\left(\left|z_{0}\right|-R\right)^{2},
\end{gathered}
$$

i.e., ||$z|-| z_{0} \| \leq R$. Thus, $(z, t)=\left(z_{1}, z_{2}, z_{3}, t\right) \in A_{R}$.

Let $s_{0} \in \mathbb{R}$ be the number from hypothesis $\left(f_{3}^{\prime}\right)$. Keeping the above notations, for a fixed $\sigma \in(0,1)$, we construct the truncation function $u_{\sigma}^{i}: \Omega_{\psi} \rightarrow \mathbb{R}$ defined by

$$
u_{\sigma}^{i}(z, t)= \begin{cases}\frac{s_{0}}{1-\sigma}\left[\left(1-\max \left(e_{i}\left(z_{1}, z_{2}, t\right), \sigma\right)\right)_{+}-\left(1-\max \left(e_{i}\left(z_{2}, z_{1}, t\right), \sigma\right)\right)_{+}\right] & \text {if } \quad n=2 i, \\ \frac{s_{0}}{(1-\sigma)^{2}}\left[\left(1-\max \left(e_{i}\left(z_{1}, z_{3}, t\right), \sigma\right)\right)_{+}-\left(1-\max \left(e_{i}\left(z_{3}, z_{1}, t\right), \sigma\right)\right)_{+}\right] \times & \\ \times\left(1-\max \left(\frac{2}{R}\left|z_{2}\right|, \sigma\right)\right)_{+}, & \text {if } \quad n \neq 2 i .\end{cases}
$$

Due to (5.11) we have the following properties: 
(p1') $\operatorname{supp} u_{\sigma}^{i}=S_{1}^{i}$;

(p2') $\left\|u_{\sigma}^{i}\right\|_{L^{\infty}} \leq\left|s_{0}\right| ;$

(p3') $\left|u_{\sigma}^{i}(x)\right|=\left|s_{0}\right|$ for every $x \in S_{\sigma}^{i}$.

Moreover, $\tilde{\tau} \# u_{\sigma}^{i}=u_{\sigma}^{i}$ for every $\tilde{\tau} \in \hat{T}_{n, i}^{\zeta_{i}}$ where the action '\#' is from (4.2). Therefore, $u_{\sigma}^{i} \in$ $H W_{0, \hat{T}_{n, i}^{\zeta_{i}}}^{1,2}\left(\Omega_{\psi}\right)$. Since $F$ is even, by using properties $\left(\mathrm{p} 1^{\prime}\right)-\left(\mathrm{p} 33^{\prime}\right)$, one has

$$
\begin{aligned}
\mathcal{F}\left(u_{\sigma}^{i}\right) & =\int_{S_{\sigma}^{i}} K(z, t) F\left(u_{\sigma}^{i}(z, t)\right) d z d t+\int_{S_{1}^{i} \backslash S_{\sigma}^{i}} K(z, t) F\left(u_{\sigma}^{i}(z, t)\right) d z d t \\
& \geq \inf _{S_{\sigma}^{i}} K \cdot F\left(s_{0}\right) \mu\left(S_{\sigma}^{i}\right)-\|K\|_{L^{\infty}} \max _{|t| \leq\left|s_{0}\right|}|F(t)| \cdot \mu\left(S_{1}^{i} \backslash S_{\sigma}^{i}\right) .
\end{aligned}
$$

On account of (5.7), (5.9), (5.12) and (5.13), if $\sigma$ is close enough to 1, the right-hand side of the latter term is positive. Thus, we can define the number

$$
\lambda_{i}^{*}=\inf \left\{\frac{\|u\|_{\nu}^{2}}{2 \mathcal{F}(u)}: u \in H W_{0, \hat{T}_{n, i}^{\zeta_{i}}}^{1,2}\left(\Omega_{\psi}\right), \mathcal{F}(u)>0\right\}<\infty .
$$

Moreover, from (5.3) and (5.4), one has that

$$
\chi_{i}=\sup \left\{\frac{2 \mathcal{F}(u)}{\|u\|_{\nu}^{2}}: u \in H W_{0, \hat{T}_{n, i}^{\zeta_{i}}}^{1,2}\left(\Omega_{\psi}\right) \backslash\{0\}\right\} \in(0, \infty)
$$

and $\chi_{i}^{-1}=\lambda_{i}^{*}$.

As in (ii), we apply Theorem 5.1 to $X=H W_{0, \hat{T}_{n, i}^{\zeta_{i}}}^{1,2}\left(\Omega_{\psi}\right)$ and to the functionals $E_{1}, E_{2}, E_{3}$ : $H W_{0, \hat{T}_{n, i}^{\zeta_{i}}}^{1,2}\left(\Omega_{\psi}\right) \rightarrow \mathbb{R}$ which are the restrictions of $\frac{1}{2}\|\cdot\|_{\nu}^{2}, \mathcal{F}$ and $\tilde{\mathcal{F}}$ to $H W_{0, \hat{T}_{n, i}^{\zeta_{i}}}^{1,2}\left(\Omega_{\psi}\right)$. Repeating a similar argument as before, we state that for $\lambda>\lambda_{i}^{*}=\chi_{i}^{-1}>0$, there exists $\delta_{i}^{\lambda}>0$ such that for each $\theta \in\left[-\delta_{i}^{\lambda}, \delta_{i}^{\lambda}\right]$, the functional $E_{1}-\lambda E_{2}-\theta E_{3}$ has at least three critical points in $H W_{0, \hat{T}_{n, i}^{\zeta_{i}}}^{1,2}\left(\Omega_{\psi}\right)$. Since $f$ and $\tilde{f}$ are odd functions, the energy functional $\mathcal{E}_{\lambda, \theta}=\frac{1}{2}\|\cdot\|_{\nu}^{2}-\lambda \mathcal{F}-\theta \tilde{\mathcal{F}}$ is even, thus $\hat{T}_{n, i}^{\zeta_{i}}$-invariant where the action of $\hat{T}_{n, i}^{\zeta_{i}}$ on $H W_{0}^{1,2}\left(\Omega_{\psi}\right)$ is given by (4.2). From the principle of symmetric criticality it follows that the critical points of $E_{1}-\lambda E_{2}-\theta E_{3}$ are also critical points for $\mathcal{E}_{\lambda, \theta}$, therefore, weak solutions for $\left(P_{\lambda, \theta}^{\nu}\right)$. Summing up the above facts, for every $i \in\left\{1, \ldots,\left[\frac{n}{2}\right]\right\}$, and for every $\lambda>\lambda_{i}^{*}$ and $\theta \in\left[-\delta_{i}^{\lambda}, \delta_{i}^{\lambda}\right]$, problem $\left(P_{\lambda, \theta}^{\nu}\right)$ has at least two distinct pairs of nonzero weak solutions $\left\{ \pm u_{i, 1}^{\lambda, \theta}, \pm u_{i, 2}^{\lambda, \theta}\right\} \subset H W_{0, \hat{T}_{n, i}^{\zeta_{i}}}^{1,2}\left(\Omega_{\psi}\right)$. A similar argument also shows (see also (ii)) that there exists $\lambda_{0}^{*}>0$ such that for every $\lambda>\lambda_{0}^{*}$ there exists $\delta_{0}^{\lambda}>0$ such that for every $\theta \in\left[-\delta_{0}^{\lambda}, \delta_{0}^{\lambda}\right]$, problem $\left(P_{\lambda, \theta}^{\nu}\right)$ has at least two distinct pairs of nonzero weak solutions $\left\{ \pm u_{0,1}^{\lambda, \theta}, \pm u_{0,2}^{\lambda, \theta}\right\} \subset H W_{0, \text { cyl }}^{1,2}\left(\Omega_{\psi}\right)$.

Now, if we choose $\Lambda^{*}=\max \left\{\lambda_{0}^{*}, \lambda_{1}^{*}, \ldots, \lambda_{\left[\frac{n}{2}\right]}^{*}\right\}$ and $\delta^{\lambda}=\min \left\{\delta_{0}^{\lambda}, \delta_{1}^{\lambda}, \ldots, \delta_{\left[\frac{n}{2}\right]}^{\lambda}\right\}$, the claim follows from Proposition 4.1 (ii)\&(iii). 


\section{Appendix: Proof of Theorem 3.1}

Although the line of the proof of Theorem 3.1 is similar to Tintarev and Fieseler [22, Proposition $4.4]$, we present its proof to make our paper self-contained. The notations and notions in our proof are taken from [22].

Let $\left\{u_{k}\right\}$ be a bounded sequence in $H W_{0, T}^{1,2}\left(G_{0}\right)$. By keeping the same notation, we naturally extend the functions $u_{k}$ to the whole group $G$ by zero on $G \backslash G_{0}$. Thus, $\left\{u_{k}\right\}$ is bounded in $H W_{0}^{1,2}(G)$ and since $T * G_{0}=G_{0}$, we also have

$$
\hat{\tau} \# u_{k}=u_{k}, \forall \hat{\tau} \in T .
$$

Since $\left(H W_{0}^{1,2}(G), D_{G}\right)$ is a dislocation pair, we may apply the abstract version of the concentration compactness principle from Tintarev and Fieseler [22, Theorem 3.1, p. 62], which guarantees the existence of a set $\mathbb{N}_{0} \subset \mathbb{N}, w^{(n)} \in H W_{0}^{1,2}(G), g_{k}^{(n)} \in D_{G}, g_{k}^{(1)}=i d$ with $k \in \mathbb{N}$, $n \in \mathbb{N}_{0}$ such that for a renumbered sequence,

$$
\begin{gathered}
w^{(n)}=w \lim g_{k}^{(n)^{-1}} u_{k} ; \\
g_{k}^{(n)^{-1}} g_{k}^{(m)} \rightarrow 0, n \neq m ; \\
u_{k}-\sum_{n \in \mathbb{N}_{0}} g_{k}^{(n)} w^{(n)} \stackrel{D_{G}}{ } 0 .
\end{gathered}
$$

Let $\eta_{k}^{(n)} \in G$ be the shifting element associated to $g_{k}^{(n)}$, see (2.2). Putting $m=1$ in (6.3), one has that $g_{k}^{(n)} \rightarrow 0(n \geq 2)$, thus $\left\{\eta_{k}^{(n)}\right\}$ has no bounded subsequence, i.e., $d_{C C}\left(0, \eta_{k}^{(n)}\right) \rightarrow \infty$ as $k \rightarrow \infty$. We claim that $w^{(n)}=0$ for every $n \geq 2$. To prove this, we distinguish two cases:

Case 1. We assume that $\mu\left(\lim \inf \left(\eta_{k}^{(n)} \circ G_{0}\right)\right)=0$. Fix $p \in G$ arbitrarily. Since the Lebesgue measure of the set $\lim \inf \left(\eta_{k}^{(n)} \circ G_{0}\right)$ is zero, we may assume that $p \notin \lim \inf \left(\eta_{k}^{(n)} \circ G_{0}\right)$. Therefore, from the definition of the Kuratowski lower-limit for sets, there exists a subsequence $\left\{\eta_{k_{j}}^{(n)}\right\}$ of $\left\{\eta_{k}^{(n)}\right\}$ such that $p \notin \eta_{k_{j}}^{(n)} \circ G_{0}$, i.e., $\eta_{k_{j}}^{(n)^{-1}} \circ p \notin G_{0}$. In particular, $u_{k_{j}}\left(\eta_{k_{j}}^{(n)^{-1}} \circ p\right)=0$. On the other hand, up to a subsequence, from (6.2) we have that the sequence $\left\{g_{k_{j}}^{(n)^{-1}} u_{k_{j}}\right\}$ converges pointwise almost everywhere to $w^{(n)}$ on $G$. Combining these facts, we obtain that

$$
w^{(n)}(p)=\lim _{j}\left(g_{k_{j}}^{(n)^{-1}} u_{k_{j}}\right)(p) \stackrel{(2.2)}{=} \lim _{j} u_{k_{j}}\left(\eta_{k_{j}}^{(n)^{-1}} \circ p\right)=0,
$$

which proves the claim.

Case 2. We assume now that $\mu\left(\liminf \left(\eta_{k}^{(n)} \circ G_{0}\right)\right)>0$. From the hypotheses $(H)_{T}^{G_{0}}$ it follows that there exists a subsequence $\left\{\eta_{k_{j}}\right\}$ of $\left\{\eta_{k}\right\}$ and a subgroup $T_{\left\{\eta_{k_{j}}\right\}}$ of $T$ with $\operatorname{card}\left(T_{\left\{\eta_{k_{j}}\right\}}\right)=\infty$ verifying relation (3.2). Assume by contradiction that $w^{(n)} \neq 0$ for some $n \geq 2$. Let $L \in \mathbb{N}$, and fix the mutually distinct elements $\hat{\tau}_{1}, \ldots, \hat{\tau}_{L} \in T_{\left\{\eta_{k_{j}}\right\}}$. It is clear that

$$
\left\|u_{k_{j}}-\sum_{l=1}^{L}\left(\hat{\tau}_{l} \# w^{(n)}\right)\left(\left(\hat{\tau}_{l} * \eta_{k_{j}}^{(n)}\right) \circ \cdot\right)\right\|_{H W(G)}^{2} \geq 0 .
$$


After the expansion of this expression, we obtain that

$$
\left\|u_{k_{j}}\right\|_{H W(G)}^{2}-2 \sum_{l=1}^{L} I_{j}^{l}+\sum_{l_{1}=1}^{L} \sum_{l_{2}=1}^{L} I_{j}^{l_{1}, l_{2}} \geq 0
$$

where

$$
I_{j}^{l}:=\left\langle u_{k_{j}},\left(\hat{\tau}_{l} \# w^{(n)}\right)\left(\left(\hat{\tau}_{l} * \eta_{k_{j}}^{(n)}\right) \circ \cdot\right)\right\rangle_{H W(G)},
$$

and

$$
I_{j}^{l_{1}, l_{2}}:=\left\langle\left(\hat{\tau}_{l_{1}} \# w^{(n)}\right)\left(\left(\hat{\tau}_{l_{1}} * \eta_{k_{j}}^{(n)}\right) \circ \cdot\right),\left(\hat{\tau}_{l_{2}} \# w^{(n)}\right)\left(\left(\hat{\tau}_{l_{2}} * \eta_{k_{j}}^{(n)}\right) \circ \cdot\right)\right\rangle_{H W(G)} .
$$

First, we have that

$$
\begin{aligned}
I_{j}^{l} & =\left\langle u_{k_{j}},\left(\hat{\tau}_{l} \# w^{(n)}\right)\left(\left(\hat{\tau}_{l} * \eta_{k_{j}}^{(n)}\right) \circ \cdot\right)\right\rangle_{H W(G)} \\
& =\left\langle u_{k_{j}}\left(\left(\hat{\tau}_{l} * \eta_{k_{j}}^{(n)}\right)^{-1} \circ \cdot\right), \hat{\tau}_{l} \# w^{(n)}\right\rangle_{H W(G)} \quad\left(\text { cf. left invariance of }\|\cdot\|_{H W(G)}\right) \\
& =\left\langle u_{k_{j}}\left(\left(\hat{\tau}_{l} * \eta_{k_{j}}^{(n)^{-1}}\right) \circ \cdot\right), \hat{\tau}_{l} \# w^{(n)}\right\rangle_{H W(G)} \quad(\mathrm{cf} .(\mathrm{TG} 2)) \\
& =\left\langle\left(\hat{\tau}_{l}^{-1} \# u_{k_{j}}\right)\left(\eta_{k_{j}}^{(n)^{-1}} \circ\left(\hat{\tau}_{l}^{-1} * \cdot\right)\right), \hat{\tau}_{l} \# w^{(n)}\right\rangle_{H W(G)} \quad(\mathrm{cf} .(\mathrm{TG} 2) \text { and }(3.1)) \\
& =\left\langle u_{k_{j}}\left(\eta_{k_{j}}^{(n)^{-1}} \circ\left(\hat{\tau}_{l}^{-1} * \cdot\right)\right), \hat{\tau}_{l} \# w^{(n)}\right\rangle_{H W(G)} \quad(\mathrm{cf} .(6.1)) \\
& =\left\langle\left(g_{k_{j}}^{(n)^{-1}} u_{k_{j}}\right)\left(\hat{\tau}_{l}^{-1} * \cdot\right), \hat{\tau}_{l} \# w^{(n)}\right\rangle_{H W(G)} \quad(\mathrm{cf} .(2.2)) \\
& =\left\langle\hat{\tau}_{l} \#\left(g_{k_{j}}^{(n)^{-1}} u_{k_{j}}\right), \hat{\tau}_{l} \# w^{(n)}\right\rangle_{H W(G)} \quad(\mathrm{cf} .(3.1)) \\
& =\left\langle\left(g_{k_{j}}^{(n)^{-1}} u_{k_{j}}\right), w^{(n)}\right\rangle_{H W(G)} \quad\left(T \text { acts isometrically on } H W_{0}^{1,2}(G), \hat{\tau}_{l} \in T\right)
\end{aligned}
$$

Therefore, according to (6.2), one has for every $l \in\{1, \ldots, L\}$ that

$$
\lim _{j} I_{j}^{l}=\lim _{j}\left\langle g_{k_{j}}^{(n)^{-1}} u_{k_{j}}, w^{(n)}\right\rangle_{H W(G)}=\left\|w^{(n)}\right\|_{H W(G)}^{2} .
$$

Now, in order to estimate $I_{j}^{l_{1}, l_{2}}$, we distinguish two cases. First, let $l_{1}=l_{2}=: l$. Since the $H W(G)$-norm is left-invariant with respect to translations and $T$ acts isometrically on $H W_{0}^{1,2}(G)$, we have

$$
I_{j}^{l, l}=\left\|w^{(n)}\right\|_{H W(G)}^{2}
$$

Second, let $l_{1} \neq l_{2}$. We claim that

$$
\lim _{j} I_{j}^{l_{1}, l_{2}}=0 .
$$

Indeed, relation (3.2) from hypothesis $(H)_{T}^{G_{0}}$, the density of $C_{0}^{\infty}(G)$ in $H W_{0}^{1,2}(G)$, as well as the Lebesgue dominance theorem imply relation (6.8). Roughly speaking, the geometrical meaning of the above phenomenon is that the compact supports of the approximating functions for $\hat{\tau}_{l_{i}} \# w^{(n)}(i=1,2)$ are far from each other after 'distant' left-translations. Now, combining relations (6.5)-(6.8), it yields

$$
\left\|u_{k_{j}}\right\|_{H W(G)}^{2} \geq L\left\|w^{(n)}\right\|_{H W(G)}^{2}+o(1) .
$$

Since $\operatorname{card}\left(T_{\left\{\eta_{k_{j}}\right\}}\right)=\infty$, then $L$ can be fixed arbitrary large, which contradicts the boundedness of $\left\{u_{k_{j}}\right\}$. Therefore, $w^{(n)}=0$. 
Consequently, in both cases we have $w^{(n)}=0$ for every $n \geq 2$. Now, from (6.4), up to a subsequence, it yields that $u_{k} \stackrel{D_{G}}{w^{(1)}}$. By using Tintarev and Fieseler [22, Lemma 9.12, p. 223], it follows that $u_{k} \rightarrow w^{(1)}$ strongly in $L^{q}(G), q \in\left(2,2_{Q}^{*}\right)$. The trivial extension of $u_{k}$ to $G \backslash G_{0}$ by zero yields that $\left.u_{k} \rightarrow w^{(1)}\right|_{G_{0}}$ strongly in $L^{q}\left(G_{0}\right), q \in\left(2,2_{Q}^{*}\right)$, which concludes the proof.

Acknowledgment. A. Kristály is grateful to the Universität Bern for the warm hospitality where this work has been initiated. Both authors thank Csaba Varga for stimulating conversations on the subject of the paper.

\section{References}

[1] A. Ambrosetti, P. H. Rabinowitz, Dual variational methods in critical point theory and applications. J. Funct. Anal. 14(1973), 349-381.

[2] Z. M. Balogh, J. T. Tyson, Polar coordinates in Carnot groups. Math. Z. 241(2002), no. 4, 697-730.

[3] I. Birindelli, E. Lanconelli, A negative answer to a one-dimensional symmetry problem in the Heisenberg group. Calc. Var. 18(2003), 357-372.

[4] T. Bartsch, M. Willem, Infinitely many nonradial solutions of a Euclidean scalar field equation. J. Funct. Anal. 117(1993), no. 2, 447-460.

[5] A. Bonfiglioli, E. Lanconelli, F. Uguzzoni, Stratified Lie groups and potential theory for their sub-Laplacian. Springer Monographs in Mathematics, Springer, Berlin, 2007.

[6] W. Y. Ding, On a conformally invariant elliptic equation on $\mathbb{R}^{n}$. Comm. Math. Phys. 107(1986), no. 2, 331-335.

[7] M. Esteban, P. L. Lions, A compactness lemma. Nonlinear Anal. 7(1983), no. 4, 381-385.

[8] G. B. Folland, E. M. Stein, Estimates for the $\bar{\partial}_{b}$ complex and analysis on the Heisenberg group. Comm. Pure Appl. Math. 27(1974), 429-522.

[9] N. Garofalo, E. Lanconelli, Existence and nonexistence results for semilinear equations on the Heisenberg group. Indiana Univ. Math. J. 41(1992), no. 1, 71-98.

[10] M. Gromov, Carnot-Carathéodory spaces seen from within. Sub-Riemannian geometry, 79323. Prog. Math. 144, Birkhäuser, Basel, 1996.

[11] J. Kobayashi, M. Ôtani, The principle of symmetric criticality for non-differentiable mappings. J. Funct. Anal. 214(2004), no. 2, 428-449.

[12] I. Kombe, Sharp weighted Rellich and uncertainty principle inequalities on Carnot grous. Communications in Applied Analysis 14(2010), no. 2, 251-272.

[13] D. Kunkle, C. Cooperman, Twenty-six moves suffice for Rubik's cube. Proceedings of the International Symposium on Symbolic and Algebraic Computation (ISSAC '07). ACM Press, 2007. 
[14] P. L. Lions, Symétrie et compacité dans les espaces de Sobolev. J. Funct. Anal. 49(1982), no. $3,315-334$.

[15] S. Maad, A semilinear problem for the Heisenberg Laplacian on unbounded domains. Canad. J. Math. 57(2005), no. 6, 1279-1290.

[16] R. Palais, The principle of symmetric criticality. Comm. Math. Phys. 69(1979), no. 1, 19-30.

[17] P. Pucci, J. Serrin, A mountain pass theorem. J. Differential Equations 60(1985), no. 1, $142-149$.

[18] B. Ricceri, A further three critical points theorem. Nonlinear Analysis 71(2009), 4151-4157.

[19] I. Schindler, K. Tintarev, Semilinear subelliptic problems without compactness on Lie groups. NoDEA Nonlinear Differential Equations Appl. 11(2004), no. 3, 299-309.

[20] W. Strauss, Existence of solitary waves in higher dimensions. Comm. Math. Phys. 55(1977), no. $2,149-162$.

[21] K. Tintarev, Semilinear elliptic problems on unbounded subsets of the Heisenberg group. Electron. J. Differential Equations 2001, No. 18, 8 pp.

[22] K. Tintarev, K.-H. Fieseler, Concentration compactness. Functional-analytic grounds and applications. Imperial College Press, London, 2007.

[23] M. Willem, Minimax theorems. Progress in Nonlinear Differential Equations and their Applications, 24. Birkhäuser Boston, Inc., Boston, MA, 1996. 